TAUP-2280-95

IASSNS-HEP-96/71

\title{
A Chiral Spin Theory in the Framework of an Invariant Evolution Parameter Formalism
}

\author{
B.Sarel \\ School of Physics and Astronomy \\ Raymond and Beveryly Sacklar Faculty of Exact Sciences \\ Tel Aviv University, 69978 Ramat Aviv, Israel \\ and \\ L.P.Horwitz* \\ School of Natural Sciences, Institute for Advanced Study \\ Princeton, N.J. 08540
}

July 27, 2021

\begin{abstract}
We present a formulation for the construction of first order equations which describe particles with spin, in the context of a manifestly covariant relativistic theory governed by an invariant evolution parameter; one obtains a consistent quantized formalism dealing with off-shell particles with spin. Our basic requirement is that the second order equation in the theory is of the Schrödinger-Stueckelberg type, which exhibits features of both the Klein-Gordon and Schrödinger equations. This requirement restricts the structure of the first order equation, in particular,
\end{abstract}

\footnotetext{
${ }^{*}$ On sabbatical leave from School of Physics and Astronomy, Raymond and Beverly Sackler Faculty of Exact Sciences, Tel Aviv University, Ramat Aviv, Israel and Department of Physics, Bar Ilan University, Ramat Gan, Israel
} 
to a chiral form. One thus obtains, in a natural way, a theory of chiral form for massive particles, which may contain both left and right chiralities, or just one of them. We observe that by iterating the first order system, we are able to obtain second order forms containing the transverse and longitudinal momentum relative to a time-like vector $t_{\mu} t^{\mu}=-1$ used to maintain covariance of the theory. This time-like vector coincides with the one used by Horwitz, Piron, and Reuse to obtain an invariant positive definite space-time scalar product, which permits the construction of an induced representation for states of a particle with spin. We discuss the currents and continuity equations, and show that these equations of motion and their currents are closely related to the spin and convection parts of the Gordon decomposition of the Dirac current. The transverse and longitudinal aspects of the particle are complementary, and can be treated in a unified manner using a tensor product Hilbert space. Introducing the electromagnetic field we find an equation which gives rise to the correct gyromagnetic ratio, and is fully Hermitian under the proposed scalar product. Finally, we show that the original structure of Dirac's equation and its solutions is obtained in the highly constrained limit in which $p_{\mu}$ is proportional to $t_{\mu}$ on mass shell. The chiral nature of the theory is apparent. We define the discrete symmetries of the theory, and find that they are represented by states which are pure left or right handed. 


\section{Introduction}

The Dirac theory of the spin $\frac{1}{2}$ particles, like the Klein-Gordon description of particles without spin, makes use of wave functions which are not localized, as pointed out by Newton and Wigner [1]. The equations of motion, although useful for describing the properties of quantum fields, are not adequate for the construction of effective oneparticle quantum theories. It has been found that the use of an invariant parameter to describe the evolution of states which are off-mass-shell provides a general framework in which this problem is solved [Q], 怔.

First order equations for particles with spin $\frac{1}{2}$, for which the evolution of the entire system is governed by an invariant parameter, have been searched for and studied by many over the years. Their importance lies in describing the behavior of the spin degrees of freedom of fermionic particles in such theories. We can trace the formulation of covariant quantum theories with an invariant evolution parameter from Fock [ब, through Stueckelberg [5], Nambu [6], Schwinger [7], and Feynman [8], up to more recent work done by Cooke [9], Horwitz and Piron [2], and Fanchi [10].

Second order equations for spin $\frac{1}{2}$ particles have been found and studied by Fock [4], Feynman [8], Horwitz et al [11], and Reuse [12]. A number of first order equations for spin $\frac{1}{2}$ particles have been proposed by Nambu [6], Feynman [8], Kubo [13], and Davidon [14]. The Dirac equation [15] does not contain an invariant evolution parameter, and applies to a three-dimensional measure space. For a summary of the subject and an extensive list of references see Fanchi [16]. Some of the first order equations were introduced in an ad hoc manner, and some of the formulations were incompatible with the postulated second order equation for the evolution of a free particle in the corresponding theories. In some cases they led to free evolution equations which are second order in the invariant parameter, admitting solutions which propagate forward and backward, thus invalidating the interpretation of the invariant parameter as an unidirectional evolution parameter [17, 18], a feature that is quite important for the interpretation of the theory.

In this paper we propose a first order equation of motion for a spin $\frac{1}{2}$ particle, in the framework of the formalism developed by Horwitz and Piron [2], which is consistent with the form of the second order evolution equation for a free particle, the SchrödingerStueckelberg equation

$$
i \partial_{\tau} \psi=\frac{P^{\mu} P_{\mu}}{2 M} \psi
$$


It seems that this kind of evolution kernel, proportional to $P^{\mu} P_{\mu}$, is best suited to describe a covariant theory (see Fanchi [19]). It leads to the correct relation for the velocities of a classical particle and permits separation of variables in the many body case [2].

We find that to achieve the goal of obtaining a first order equation for a spin $\frac{1}{2}$ particle, it is necessary to introduce nilpotent operators which result in a chiral theory even though we are not restricted to massless fermions. This result may be relevant to the present situation in weak interactions and neutrino physics, where one finds chiral fields although it is not clear the neutrino mass is zero, and when theories beyond the standard model are considered [20, 21, 22]. We shall restrict our study of interactions here, however, to the case of the $\mathrm{U}(1)$ electromagnetic gauge.

The general outline of this work is as follows. In Section II we present a short summary of the basics of the formalism we use. We then establish our basic requirements of the equation, and the logic in deriving it. In Section III we define the basic structure of the equations of motion, the form of a continuity equation, currents, and probability density. The basic requirements force us to seek nilpotent matrices as building blocks for the equation, so we investigate all possible nilpotent $4 \times 4$ matrices in Appendix A, (and state some useful facts about them), and we state the Lorentz covariant appropriate nilpotent forms. We observe that it is not possible to construct a single SchrödingerStueckelberg type equation by iteration. We manage however, in Section IV, to construct two equations of motion, for transverse and longitudinal modes. After doing that, we still have four possible forms for both versions, and we show the relations among them. Two of them vary by the relative signs of their chiral components, and the other two are complementary in a sense that is described later on. In Section V, we investigate the form of the resulting currents and establish the validity of the form of the probability density for both versions. At this stage we observe the connection between the transverse and longitudinal equations, and the spin and convection currents, respectively, of the Gordon decomposition of the Dirac current. In Section VI we define the tensor product Hilbert space over the longitudinal and transverse modes, and show that our formulation is fully compatible with the one of Horwitz and Arshansky [23]. Next, in Section VII, we introduce a minimal coupling for the gauge field, producing electromagnetic interaction. We discuss the second order form of the generator of motion, and show it coincides with the one achieved by Horwitz and Arshansky [23]. It implies the correct gyromagnetic 
ratio, and is fully Hermitian under the positive definite, invariant scalar product for the quantum mechanical Hilbert space. In Section VIII, we show that we recover the original Dirac equation and its solutions in the limit where $p_{\mu}$ is constrained to be proportional to $t_{\mu}$ on mass shell. The solutions of the equations of motion are discussed in Appendix B. We explore the discrete symmetries of the theory, in Appendix Q, and define the generalized parity, charge conjugation, and $\tau$ reversal transformations. The states that are transformed one into the other under these transformations are pure left or right handed. Finally, in Appendix D we make explicit the representation of the Dirac matrices we used for calculations, which lends itself to the problem, the chiral representation.

We use the notation

$$
x^{\mu} \equiv\left(x^{0}, x^{1}, x^{2}, x^{3}\right)
$$

and we shall always use the symbol $x^{0}$ as the time component, and reserve $t^{\mu}$ for the time-like vector. We use an opposite metric relative to the Bjorken-Drell convention [24]

$$
g_{00}=-1=-g_{11}=-g_{22}=-g_{33}
$$

Furthermore, we shall use the notation

$$
(\gamma \cdot v) \equiv\left(\gamma^{\mu} v_{\mu}\right)
$$

where $v_{\mu}$ is a four-vector. We also use the uppercase $P_{\mu}$ to denote an operator, and lowercase $p_{\mu}$ for eigenvalues.

\section{II. $\tau$-formalism}

We refer to the invariant parameter as $\tau$, the invariant universal world time, which describes the evolution of an event moving through space-time. The notion of the need of some invariant evolution parameter to replace the covariant time is not new, and we give some of the arguments as presented in 25].

Non-relativistic quantum mechanics uses the Newtonian universal time to describe a state in terms of square integrable functions over three-space at a specific time, which evolve according to Schrödinger's equation. But the Hilbert spaces associated with different times are distinct; we cannot superpose wave functions at different times. This 
situation is inconsistent with special relativity. Viewed from some other frame the wave function becomes a function on different times, thus losing its interpretation as a state.

The Klein-Gordon and Dirac equations have been able to resolve the problems of covariance; they have a manifestly covariant form. But the problem of constructing localized states still remains. The inconsistency of the solutions of the Klein-Gordon equation as an amplitude for a local probability density was shown by Newton and Wigner [怔]. They showed that the distribution corresponding to a localized particle is an eigenfunction of the operator

$$
X_{N W}=i\left(\frac{\partial}{\partial \mathbf{p}}-\frac{\mathbf{p}}{2 E^{2}}\right)
$$

and the wave function corresponding to a localized particle is spread out by the order of a Compton wavelength. They reached similar conclusions concerning the Dirac equation. Hegerfeldt [26], has shown that a distribution at a specific time, defined to have compact support, or localized in some other sense, does not maintain its localizability, and evolves out of the light cone, i.e. acausally.

Quantum field theories make the transformation laws of special relativity and quantum mechanics consistent by assigning the spatial variables to the same parametric role as the time. The dynamical variables are operator valued fields which are functions on this parametric space-time. But the one particle sector wave functions of such theories describing the transition amplitudes between the vacuum and one particle states, suffers from the same difficulties as mentioned above. Predictions of phenomena concerning local properties in space-time are very difficult to formulate and interpret (e.g. interference phenomena), although spectral properties of non-local observables (energy, Lamb shift, anomalous magnetic moment), can be computed and are in excellent agreement with experiment.

The basic difficulty of developing a consistent theory which incorporates the ideas of special relativity and quantum mechanics is related to questions concerning the relationship between time and locality. On one hand, space and time transform with the Lorentz group (and thus this relativistic time has a geometrical interpretation), and on the other hand, in a specific frame, it has been considered as a measure of evolution, of change. A way to resolve this ambiguity is to define the state of a system in terms of a distribution of events in space and time, while their evolution is parameterized by the time indicated on an ideal clock which is associated with every inertial frame (see 
[27 for further discussion). We call this parameter the universal time $\tau$, and it can be identified with Newton's time.

Stueckelberg [5], Horwitz and Piron [2], and others [7, 8, 10] developed an underlying formalism incorporating the invariant parameter $\tau$ which enabled them to construct a consistent manifestly covariant relativistic classical and quantum theory. The NewtonWigner [2] as well as the Landau-Peierls [28] problems have been understood in this framework. Two body problems, both for bound states and scattering have been treated, and the Zeeman [29] effect and selection rules [30] for radiation worked out. The spinless theory, in interaction with radiation (U(1) gauge field) has been second quantized [31. We state briefly the main principles of the formalism.

The equations of motion of the classical theory, may be derived from the Hamilton principle [2]

$$
\delta \int\left(p_{\mu} d q^{\mu}-K\left(p^{\mu}, q^{\mu}\right) d \tau\right)=0
$$

This principle is equivalent to the canonical equations

$$
\begin{aligned}
\frac{d p_{\mu}}{d \tau} & =-\frac{\partial K}{\partial q^{\mu}} \\
\frac{d q^{\mu}}{d \tau} & =\frac{\partial K}{\partial p_{\mu}}
\end{aligned}
$$

describing the motion of an "event" along its world line (trajectory). For example, for a free event one takes

$$
K_{0}=\frac{p^{\mu} p_{\mu}}{2 M}=\frac{\mathbf{p}^{2}-E^{2}}{2 M}
$$

where $M$ is a given property of the event, and sets the scale between $\tau$ and the quantities of motion. We then have

$$
\frac{d \mathbf{x}}{d \tau}=\frac{\mathbf{p}}{M} \quad ; \quad \frac{d t}{d \tau}=\frac{E}{M}
$$

The proper time interval for the motion of a free event is defined by

$$
d s^{2}=d t^{2}-d \mathbf{x}^{2}
$$

and satisfies

$$
d s^{2}=\frac{m^{2}}{M^{2}} d \tau^{2}
$$

where $m^{2}=E^{2}-\mathbf{p}^{2}$ is a dynamical variable to be determined by initial conditions and dynamics of the system. If initial conditions are chosen so that $m^{2}=M^{2}$, the 
"on-shell" condition, then the proper time interval and the universal world time $\tau$, coincide. It should be noted that the theory is not constrained to time-like motion, so tachyonic propagation of events is possible. However, this does not imply explicitly the existence of tachyonic particles in laboratory measurements since one generally observes asymptotic states as the initial and final outcome of collision experiments. Due to the asymptotic conservation of the generator of free propagation $\frac{P^{\mu} P_{\mu}}{2 M}$, for example, in potential scattering, states that are initially in time-like motion are time-like in the asymptotic final state as well. The structure of the theory, however, does not exclude tachyons a priori, classically or quantum mechanically.

In the quantum domain, the states of the system for a given $\tau$ are described in the Hilbert space $L^{2}\left(R^{4}, d^{3} x d t\right)$, the space of square integrable functions of four variables given for the spinless case as $(\psi, \chi)=\int \psi^{*} \chi d^{4} x$. We do not define the scalar product for spin $\frac{1}{2}$ particles at this stage; instead we shall show that we obtain the scalar product suggested by Horwitz and Arshansky [23, and Arensburg and Horwitz [32, from considerations of the equation of continuity.

The observables of spacetime coordinates and momenta satisfy the commutation relations

$$
i\left[P^{\mu}, Q^{\nu}\right]=g^{\mu \nu} \mathbf{1}
$$

The evolution of a state vector is described by the Schrödinger type equation

$$
i \partial_{\tau} \psi=K \psi
$$

where for a free particle $K=\frac{P^{2}}{2 M}$, resulting in the Schrödinger-Stueckelberg equation (11).

Horwitz and Arshansky [23] have suggested a second order equation for particles with spin $\frac{1}{2}$. They argue as follows. For a particle with spin the components of the wave function must transform as a representation of the Lorentz group. The norm must be invariant, so the representation must be unitary. But the Lorentz group is a non-compact group, therefore the unitary representations are infinite dimensional, containing all spins; such a ladder representation would, however, introduce problems with the application of the Pauli principle, for example, in the Sommerfeld model of a metal. If one were to use an induced representation based on the particle four-momentum as done by Wigner [33, the expectation value of $x^{\mu}$, which by Eq. (9) is replaced by $i \frac{\partial}{\partial p^{\mu}}$, would not be 
covariant (the derivative acts on the unitary operator of the little group defined by $p^{\mu}$ ). Their solution consisted of introducing a representation induced on the little group of a unit time-like vector, which we denote here by $t^{\mu}$, which commutes with $x^{\mu}$ and $p^{\mu}$. They described the transformation properties of the wave function, and found the form of the positive definite, covariant norm to be

$$
N=\int d^{4} x \bar{\psi}_{\tau t}(x)(\gamma \cdot t) \psi_{\tau t}(x)
$$

where $\psi_{\tau t}(x)$ is the Dirac spinor, and $\gamma^{\mu}$ the usual Dirac matrices. They constructed the Hermitian and anti-hermitian parts of the operator $(\gamma \cdot P)$ under the scalar product associated with the norm. These are, in Hermitian form (under the norm (11)),

$$
\begin{aligned}
& K_{L}=\frac{1}{2}((\gamma \cdot P)+(\gamma \cdot t)(\gamma \cdot P)(\gamma \cdot t))=-(P \cdot t)(\gamma \cdot t) \\
& K_{T}=\frac{1}{2} \gamma^{5}((\gamma \cdot P)-(\gamma \cdot t)(\gamma \cdot P)(\gamma \cdot t))=-2 i \gamma^{5}(P \cdot K)(\gamma \cdot t)
\end{aligned}
$$

where $K^{\mu}=\Sigma^{\mu \nu} t_{\nu}, \Sigma^{\mu \nu}=\frac{1}{4} i\left[\gamma^{\mu}, \gamma^{\nu}\right]$, and the subscripts $\mathrm{T}$ and $\mathrm{L}$ denote transverse and longitudinal parts relative to the time-like vector $t_{\mu}$. Since

$$
K_{L}^{2}=(P \cdot t)^{2} \quad ; \quad K_{T}^{2}=P^{2}+(P \cdot t)^{2}
$$

for the equation of evolution, Eq. (10), one can write

$$
i \partial_{\tau} \psi=\frac{1}{2 M}\left(K_{T}^{2}-K_{L}^{2}\right) \psi
$$

By introducing minimal coupling $P_{\mu} \rightarrow P_{\mu}-e A_{\mu}$ they then obtained

$$
i \partial_{\tau} \psi=\frac{(P-e A)^{2}}{2 M} \psi+\frac{e}{2 M} \Sigma_{t}^{\mu \nu} F_{\mu \nu} \psi
$$

where

$$
\Sigma_{t}^{\mu \nu}=\Sigma^{\mu \nu}+K^{\mu} t^{\nu}-K^{\nu} t^{\mu}
$$

This equation reproduces the correct gyromagnetic ratio, and does not contain the nonhermitian spin term appearing in Dirac's electromagnetically coupled, second order equation (in the special frame $t_{\mu}=(1,0,0,0)$, one easily sees that $\Sigma^{0 j}$ is canceled so that there is no direct coupling of the electric field with spin in this special frame). Note that $\Sigma^{\mu \nu} F_{\mu \nu}$, appearing in the Dirac second order equation, contains $i \sigma \cdot \mathbf{E}$ as well as 
$\sigma \cdot \mathbf{H}$; the former is not Hermitian in the norm $\int \psi^{*}(x) \psi(x) d^{3} x$, which is Dirac's scalar product.

In a later work, Arensburg and Horwitz [32] extended the formalism to a first order equation for spin $\frac{1}{2}$. Since the $K_{T}$ part is responsible for the production of the correct gyromagnetic ratio, they postulated a first order equation of the form

$$
i \partial_{\tau} \psi=2\left(P \cdot K_{T}\right)(\gamma \cdot t) \psi
$$

(Their $K_{T}$ is equivalent to that of Ref. [23] up to a factor $-i \gamma^{5}$ ). Furthermore, they found its solutions and associated current, and showed that the current, although exhibiting a space-like nature at each point on the orbit (defined by $t_{\mu}$ ), integrated over all possible $t_{\mu}$ in the forward light cone (completing the natural scalar product of an induced representation), reduces to a time-like current vector.

Eq. (18) does not, however, conform to the Schrödinger-Stueckelberg equation (1) by iteration; it leads to a second order equation in $\tau$. This is our original motivation in

trying to obtain a new kind of first order equation for spin $\frac{1}{2}$ particles in this framework.

\section{General features of the equations}

\subsection{Basic structure}

In our attempt to find a manifestly covariant equation for an event with spin we require some general features of the equation concerning the relation between first and second order equations, namely the Dirac equation and the Klein-Gordon equation. We proceed from the point of view that a first order equation is an additional condition on the second order one, while at the same time introducing the notion of spin. In the process we narrow down the number of available options and maintain only the suitable ones.

The desired equation should contain first order derivatives only, giving equal footing to the treatment of space and time. Another requirement is that the equation be Lorentz covariant, i.e., we wish the equation to be form covariant in respect to the choice of inertial frames. We assume that the space-time derivatives are coupled to an object constructed from $\gamma^{\mu}$ matrices and perhaps some other four-vectors, and work with Dirac spinors, since we want the theory to be as close as possible to the standard theory of Dirac. For the actual Lorentz transformations of the wave functions and equations we 
use the well known form [24, 34] (this form is also applicable to the more general case treated in [11], 23]; we use $\sigma^{\mu \nu}$ in place of $\Sigma^{\mu \nu}$ henceforth for notational simplicity)

$$
S(\Lambda)=e^{-\frac{i}{4} \sigma^{\mu \nu} \omega_{\mu \nu}}
$$

where

$$
\sigma^{\mu \nu}=\frac{i}{2}\left[\gamma^{\mu}, \gamma^{\nu}\right]
$$

and $\omega_{\mu \nu}$ are the antisymmetric transformation parameters.

We also require the equation to conform to a form of the Schrödinger-Stueckelberg equation by iteration, thus ensuring the free solutions of the spin particle to be also solutions of that form of Schrödinger-Stueckelberg equation, in parallel to the relation between Dirac's equation and the Klein-Gordon equation. This way each component of the wave function satisfies the free form of the Schrödinger-Stueckelberg equation separately. From another point of view we may regard the desired equation as an additional condition on the solutions of the Schrödinger-Stueckelberg equation, as the Dirac equation is to those of the Klein-Gordon equation.

Now we postulate the most general form of a first order spin equation

$$
L(P) \psi=s_{-} N_{-} i \partial_{\tau} \psi+s_{+} M N_{+} \psi
$$

where $L(P)$ is a linear function of first order space-time derivatives, namely a function of $P_{\mu}$, and $s_{+}$and $s_{-}$are sign variables to be determined. $N_{+}$and $N_{-}$are unknown matrices at this stage. The second term on the R.H.S. of Eq. (21) has no derivatives. To compensate for the dimensional deficiency we introduce the scale factor $\mathrm{M}$, which appears in the Schrödinger-Stueckelberg equation. This term is necessary for maintaining the first order derivative in respect to $\tau$, after the iteration is done.

To get the Schrödinger-Stueckelberg equation we multiply by $L(P)$ on the left

$$
L^{2}(P) \psi=s_{-} L(P) N_{-} i \partial_{\tau} \psi+s_{+} M L(P) N_{+} \psi
$$

Now, let us define the commutators and anticommutators for the operators in Eq. (21)

$$
\begin{aligned}
\tilde{C}_{l+} & \equiv\left\{L(P), N_{+}\right\} \\
\tilde{C}_{l-} & \equiv\left\{L(P), N_{-}\right\} \\
\tilde{C}_{n n} & \equiv\left\{N_{+}, N_{-}\right\}
\end{aligned}
$$




$$
\begin{aligned}
C_{l+} & \equiv\left[L(P), N_{+}\right] \\
C_{l-} & \equiv\left[L(P), N_{-}\right] \\
C_{n n} & \equiv\left[N_{+}, N_{-}\right]
\end{aligned}
$$

We substitute Eq. (21) into Eq. (22) to obtain

$$
L^{2}(P) \psi=N_{-}^{2} \partial_{\tau}^{2} \psi-s_{+} s_{-} M \tilde{C}_{n n} i \partial_{\tau} \psi+s_{-} \tilde{C}_{l-} i \partial_{\tau} \psi+s_{+} M \tilde{C}_{l+} \psi-M^{2} N_{+}^{2} \psi
$$

We do not want second order derivatives in respect to $\tau$, so we must have

$$
N_{-}^{2}=0
$$

Since we postulated that only first order space-time derivatives appear in $L(P)$, and since Eq. (24) is supposed to coincide with the Schrödinger-Stueckelberg equation (11), we require in addition to Eq. (25) also that

$$
\tilde{C}_{l+}=\tilde{C}_{l-}=0
$$

This gets rid of first order space-time derivatives in Eq. (24), and leaves only second order ones in $L^{2}(P)$. We still have an unwanted term, $M^{2} N_{+}^{2} \psi$, and we require it to be zero (we could alternatively absorb it in the phase of the wave function). Thus, we obtain

$$
\left[-s_{+} s_{-} M \tilde{C}_{n n}\right]^{-1} L^{2}(P) \psi_{(\tilde{c})}=i \partial_{\tau} \psi_{(\tilde{c})}
$$

which is of the form of the desired equation (1), if we can find a solution for the conditions

$$
L^{2}(P)= \pm P^{2} ; \tilde{C}_{n n}= \pm 2
$$

The subscript ( $\tilde{c})$ in Eq. (27) represents an equation derived using anticommutators.

We may use commutators instead of anticommutators, and repeating the previous procedure we reach the same conclusions concerning $N_{+}, N_{-}$and $\tilde{C}_{n n}$. However, this time there is the requirement that

$$
C_{l+}=C_{l-}=0
$$

The equation analogous to Eq. (27) is

$$
\left[s_{+} s_{-} M \tilde{C}_{n n}\right]^{-1} L^{2}(P) \psi_{(c)}=i \partial_{\tau} \psi_{(c)}
$$

The subscript $(c)$ represents an equation derived using commutators. As we see later on, we shall need both forms. In addition, we require that the equation have a continuity equation, conserved positive definite probability, and currents. 


\subsection{Continuity equation and currents}

We are interested in achieving a continuity equation for Eq. (21) of the form

$$
\partial_{\alpha} j^{\alpha}=0 ; \quad(\alpha=0,1,2,3, \tau)
$$

in order to obtain the usual interpretation, where $j^{\tau}=\rho$ should be the probability density, conserved through Eq. (31). The simple way to do this is clearly shown in all textbooks [24, 34, 35] for the Dirac equation, and we follow this general method. We observe that since $N_{-}$is nilpotent, the probability density cannot be of the usual Dirac form $\psi^{\dagger} \psi$, but contains a matrix between $\psi^{\dagger}$ and $\psi$ (a nilpotent does not have an inverse so there is no way to get a pure $i \partial_{\tau} \psi$ term in Eq. (21)). Refs. [23, 32] show that the scalar product for the second order equation obtained from Eq. (11) is

$$
\left(\phi_{\text {Dirac }}, \psi_{\text {Dirac }}\right)=\int d^{4} p \bar{\phi}_{\text {Dirac }}(\gamma \cdot t) \psi_{\text {Dirac }}
$$

where $t_{\mu}$ is the same time-like four-vector with norm $t^{2}=-1$ mentioned earlier. The time-like nature of $t_{\mu}$ is crucial for the scalar product to be positive definite. [Of course, $\phi, \psi$ depend on $t^{\mu}$, so that (32) is a covariant structure on a bundle (i.e., as an induced representation).] The probability density is just the special case of the integrand of the scalar product when $\phi=\psi$. To find the continuity equation one multiplies the equation from the left by $\psi^{\dagger} \gamma^{0}$ to get

$$
\bar{\psi} L(P) \psi=s_{-} \bar{\psi} N_{-} i \partial_{\tau} \psi+s_{+} M \bar{\psi} N_{+} \psi
$$

then multiply the conjugated equation from the right by $\gamma^{0} \psi$ to get

$$
\psi^{\dagger} L^{\dagger}(-\overleftarrow{P}) \gamma^{0} \psi=-s_{-} \psi^{\dagger} i \stackrel{\overleftarrow{\partial_{\tau}}}{N_{-}^{\dagger}} \gamma^{0} \psi+s_{+} M \psi^{\dagger} N_{+}^{\dagger} \gamma^{0} \psi
$$

and subtract Eq. (34) from Eq. (33). In order to achieve the form (31) we must have

$$
\begin{aligned}
\gamma^{0}\left(N_{+}\right)^{\dagger} \gamma^{0} & =N_{+} \\
\gamma^{0}\left(N_{-}\right)^{\dagger} \gamma^{0} & =N_{-} \\
\gamma^{0}\left(L^{\dagger}(-\overleftarrow{P})\right) \gamma^{0} & =-L(\overleftarrow{P})
\end{aligned}
$$

so that we get

$$
\bar{\psi}(L(\vec{P})+L(\overleftarrow{P})) \psi=s_{-} \bar{\psi}\left(N_{-}\left[i \stackrel{\overleftarrow{\partial}}{\partial_{\tau}}+i \overrightarrow{\partial_{\tau}}\right]\right) \psi
$$


and finally

$$
\partial_{\mu}\left(\bar{\psi} b^{\mu} \psi\right)=s_{-} \partial_{\tau}\left(\bar{\psi} N_{-} \psi\right)
$$

where it is assumed that the form of $L(P)$ is $b^{\mu} i \partial_{\mu}$, and $b^{\mu}$ is some yet unspecified object with a Lorentz index which couples to $P_{\mu}$. We must therefore now search for nilpotents for which $\left(\bar{\psi} N_{-} \psi\right)$ is positive. A general analysis is carried in Appendix $\mathrm{A}$.

\subsection{Acceptable nilpotents}

One finds (in Appendix $\mathrm{A}$ ) that the candidates for nilpotents which pertain to a positive definite probability density are:

$$
\begin{array}{ll}
\left(\gamma \cdot l^{( \pm)}\right) & :(\gamma \cdot t)\left(\mathbf{1} \pm \gamma^{5}\right) \\
\left(\gamma \cdot l^{( \pm)}\right) & : \quad(\gamma \cdot t) \pm \sigma t s \\
(\gamma \cdot t) \pm i \gamma^{5} & : \quad(\gamma \cdot t) \pm \sigma t s
\end{array}
$$

where we denote

$$
\sigma t s \equiv \sigma^{\mu \nu} t_{\mu} s_{\nu}
$$

and $l, s$, and $t$ are light-like, space-like, and time-like vectors respectively.

Nilpotents of this type come in non-equivalent pairs, in the sense that no unitary transformation connects the members of the pair (see Appendix A). Furthermore, pairs in the columns of Eqs. (38) may be equivalent since we are able to transform from one to another through a unitary transformation. In any case we look at all four possibilities in the quest for $L(P)$. Each nilpotent pair can be seen to be formed of two parts, a projection operator $P r_{ \pm}$, and $(\gamma \cdot t)$, such that $(\gamma \cdot t) \cdot P r_{-}=P r_{+} \cdot(\gamma \cdot t)$. In particular,

$$
\begin{array}{ll}
\left(\gamma \cdot l^{( \pm)}\right) & =(\gamma \cdot t)(\mathbf{1} \pm(\gamma \cdot t)(\gamma \cdot s)) \\
(\gamma \cdot t) \pm i \gamma^{5} & =(\gamma \cdot t)\left(\mathbf{1} \pm i(\gamma \cdot t) \gamma^{5}\right) \\
(\gamma \cdot t) \pm(\gamma \cdot t) \gamma^{5} & =(\gamma \cdot t)\left(\mathbf{1} \pm \gamma^{5}\right) \\
(\gamma \cdot t) \pm \sigma t s & =(\gamma \cdot t)(\mathbf{1} \pm i(\gamma \cdot s))
\end{array}
$$

The last of Eqs. (40) is due to the fact that $(t \cdot s)=0$ and $\sigma t s=\frac{i}{2}[(\gamma \cdot t),(\gamma \cdot s)]$. 


\subsection{The four-momentum part : $L(P)$}

We wish $L(P)$ to be linear in first derivatives, have zero anticommutators with $N_{ \pm}$(see Eq. (26)), produce the four-current part of the continuity equation, iterate to $\pm P^{2}$, and be Lorentz covariant. We check the four available forms of nilpotents in Eqs. (38), stemming from the specific reference frame in which they were found. To comply with Eq. (26) we search for all $\Gamma$ 's (see Appendix A) which anticommute with $N_{ \pm}$, then we couple $P_{\mu}$ in all possible ways, and check the anticommutators, and other criteria. The same procedure must be repeated for commutators of Eq. (29). Going through all options of Eq. (40) is a tedious process, and we shall demonstrate only an example. Let us partly analyze the nilpotent form of $N_{ \pm} \equiv\left(\gamma \cdot l^{( \pm)}\right)$. As the original non-covariant form we take $\gamma^{0} \pm \gamma^{1}$. The $\Gamma^{\prime}$ 's that anticommute with $\gamma^{0}=\Gamma_{2}$ and $\gamma^{1}=-i \Gamma_{3}$ are :

$$
\Gamma_{4}, \Gamma_{5}, \Gamma_{6}, \Gamma_{16} \Longleftrightarrow i \gamma^{2}, i \gamma^{3}, \gamma^{0} \gamma^{1}, \gamma^{5}
$$

Since it is required that $L^{2}(P)$ must be a multiple of the identity matrix, if we wish to put in any combination of the matrices appearing in Eq. (41), we must divide them into two sets, for which the matrices in a set anticommute among themselves, and commute with all other matrices of the other set, and consider each set separately. Otherwise, after the iteration of $L(P)$ we would find non-diagonal parts due to the anticommutator of two commuting matrices. The two sets are $\left(\gamma^{0} \gamma^{1}\right)$ and $\left(i \gamma^{2}, i \gamma^{3}, \gamma^{5}\right)$. For example, the combination of $\gamma^{0} \gamma^{1}$ can be coupled to $P^{\mu}$ in a covariant way as

$$
\gamma^{0} \gamma^{1} P_{0} v_{1} \pm \gamma^{0} \gamma^{1} P_{1} v_{0} \rightarrow \sigma P v
$$

where $P_{\mu}=\left(P_{0}, P_{1}, 0,0\right)$, and $v=\left(v_{0}, v_{1}, 0,0\right)$ is some vector, in this frame (since there are two matrices we form a tensor). If we want to conform to Eq. (26) by taking $\left(\gamma \cdot l^{( \pm)}\right)$ as $N_{+}, N_{-}$and we do not want $P_{\mu}$ to be only light-like, then $v$ must be light-like (since $\tilde{C}_{l \pm}=\left\{\sigma P v, \gamma \cdot l^{( \pm)}\right\}=0$ implies $P \propto l^{( \pm)}$or $\left.v \propto l^{( \pm)}\right)$. But then the vector $l^{( \pm)}$must be the same in both $N_{+}, N_{-}$(either $l^{(+)}$or $\left.l^{(-)}\right)$, which makes $\tilde{C}_{n n}=0$. This contradicts our basic assumptions, so this sub-form must be discarded.

We have required in Section III that the iteration of the first order equation should give us $L^{2}(P)= \pm P^{2}$. Checking all available options of the nilpotents of Eq. (40), indicates that we are unable to get such a form by iteration (at least not under our initial assumptions concerning the iteration process). [In Section VIII we shall show how to 
produce $P^{2}$ by a somewhat different iteration procedure (obtained in a rigid limiting case); in that procedure we lose the Hermiticity of the spin term in the second order electromagnetically coupled equation, getting an expression as in the Dirac equation. Furthermore, we lose the structure of the continuity equation.] This applies to commutators and anticommutators alike. Nevertheless we proceed, considering the transverse and longitudinal options separately, combining them in Section VI. We start by choosing from the anticommutator forms. From them we choose

$$
N_{ \pm}=(\gamma \cdot t)\left(\mathbf{1} \pm \gamma^{5}\right)
$$

and

$$
L_{\perp}(P)=\sigma^{\mu \nu} P_{\mu} t_{\nu}
$$

This is because in doing so, we deal with only one additional four-vector, $t_{\mu}$, while retaining the ability to achieve the gyromagnetic ratio, and exhibiting some features concerning chirality due to the use of the projection operators $\frac{1}{2}\left(\mathbf{1} \pm \gamma^{5}\right)$. It also seems that this is the simplest choice which has as many benefits as possible in this situation. As we show later on, this choice gives rise to the transverse Schrödinger-Stueckelberg equation. Not excluding the existence of a longitudinal equation also, we follow our current choice for $N_{ \pm}$, and take the simplest choice for the longitudinal equation, namely

$$
L_{\|}(P)=i(P \cdot t)
$$

\section{The equations of motion}

We now discuss the consequences of the above choice for the forms of $N_{ \pm}$and $L(P)$ 's. This discussion concerns what we call the transverse and longitudinal SchrödingerStueckelberg equations.

\subsection{Transverse equations of motion}

We have

$$
\tilde{C}_{n n}=\left\{(\gamma \cdot t)\left(\mathbf{1}-\gamma^{5}\right),(\gamma \cdot t)\left(\mathbf{1}+\gamma^{5}\right)\right\}=-4 t^{2}
$$

taking $t^{2}=-1$, a unit time-like four-vector, we have to arrange an additional factor of 2 to get the desired form of $\frac{1}{2 M}$. We place this factor with $N_{+}$. [In Section VIII we show 
how to get the solutions of the Dirac equation in the limit where $p_{\mu}$ and $t_{\mu}$ coincide. For that to happen we have to take the factor 2 as indicated.] The anticommutators satisfy of course

$$
\left\{(\gamma \cdot t)\left(\mathbf{1} \pm \gamma^{5}\right),(\sigma P t)\right\}=0
$$

which follows from the important relation

$$
\{(\gamma \cdot t),(\sigma P t)\}=0
$$

Iterating $L_{\perp}(P)$ we obtain

$$
(\sigma P t)^{2}=P^{2} t^{2}-(P \cdot t)^{2}
$$

We define the transverse and longitudinal momentum, relative to $t_{\mu}$, as

$$
\begin{aligned}
P_{\perp \mu} & =P_{\mu}+(P \cdot t) t_{\mu} \\
P_{\| \mu} & =-(P \cdot t) t_{\mu}
\end{aligned}
$$

and we therefore have

$$
(\sigma P t)^{2}=t^{2} P_{\perp}^{2}=-P_{\perp}^{2}
$$

The equation of motion is

$$
-\hbar c\left(\sigma^{\mu \nu} i \partial_{\mu} t_{\nu}\right) \psi_{\perp}=\hbar s_{-}(\gamma \cdot t)\left(\mathbf{1}-\gamma^{5}\right) i \partial_{\tau} \psi_{\perp}+s_{+} \frac{M c^{2}}{2}(\gamma \cdot t)\left(\mathbf{1}+\gamma^{5}\right) \psi_{\perp}
$$

We display $\hbar$ and $c$ in this principal equation; elsewhere we take $\hbar=c=1$. It is understood that $x^{0} \equiv$ ct. Notice that in our metric $P_{\mu}=-i \frac{\partial}{\partial x^{\mu}}$.

Iterating, we obtain

$$
\frac{P_{\perp}^{2}}{s_{+} s_{-} 2 M} \psi_{\perp}=i \partial_{\tau} \psi_{\perp}
$$

This forces the signs (with our choice of phase) to be either both positive or both negative, so that $s_{+} s_{-}=1$, and we get the transverse Schrödinger-Stueckelberg equation

$$
\frac{P_{\perp}^{2}}{2 M} \psi_{\perp}=i \partial_{\tau} \psi_{\perp}
$$

which we assume to hold. Using the notation of Horwitz and Arshansky [23, $L^{2}(P)=-K_{T}^{2}$, which generates the evolution of transverse momentum only. Note that $\psi_{\perp}$ contains only the transverse space-time coordinates. 
We have not excluded the possibility of a longitudinal Schrödinger-Stueckelberg equation; from another, independent, first order equation, which we shall discuss later, we shall also have

$$
\frac{P_{\|}^{2}}{2 M} \psi_{\|}=i \partial_{\tau} \psi_{\|}
$$

where $\psi_{\|}$contains only longitudinal space-time coordinates. The subscripts $\perp$ and $\|$ are introduced for making a distinction between the wave functions of the transverse and longitudinal equations. These indeed constitute kinematically independent degrees of freedom.

Since Eq. (52) contains projectors we can decompose it into two coupled equations by multiplying it from the left with the same projectors. Denoting the wave function as composed of two chiral spinors, (two-component spinors in the chiral representation),

$$
\psi_{L}=\frac{1}{2}\left(\mathbf{1}-\gamma^{5}\right) \psi \quad \psi_{R}=\frac{1}{2}\left(\mathbf{1}+\gamma^{5}\right) \psi
$$

we obtain

$$
\begin{aligned}
& (\gamma \cdot t)(\sigma P t) \psi_{\perp L}=s_{+} M \psi_{\perp R} \\
& (\gamma \cdot t)(\sigma P t) \psi_{\perp R}=2 s_{-} i \partial_{\tau} \psi_{\perp L}
\end{aligned}
$$

This is an explicit chiral decomposition, not symmetric for left and right handed spinors, a matter discussed later on. Such a form for equations of the first order in $\tau$-formalism has been proposed by Davidon [14], in a somewhat ad hoc manner. He could not overcome the problem of finding a positive definite probability density, and he obtained a non-hermitian spin term after coupling the electromagnetic field. The equations (56),(57) are completely equivalent to the one equation (52); it is just a rewriting.

Concerning the sign variables $s_{+}$and $s_{-}$, it seems that we have two choices. Denoting by $\psi_{\perp+}$ and $\psi_{\perp_{-}}$the solutions of the two sign versions of Eq. (52), by adding and subtracting the two versions, we get exactly the equations (56),(57), with the following relation between $\psi_{\perp+}, \psi_{\perp-}$ and $\psi_{\perp L}, \psi_{\perp R}$

$$
\begin{aligned}
& \psi_{\perp+}+\psi_{\perp-}=\psi_{\perp R} \\
& \psi_{\perp+}-\psi_{\perp-}=\psi_{\perp L}
\end{aligned}
$$


As can be seen from Eq. (37), the sign $s_{-}$(equal to $s_{+}$in our treatment), sets the sign of the so called $g_{\tau \tau}$ component of the metric.

After we have discussed the two options of sign assignment, we cannot ignore the two possibilities of nilpotent assignment. Instead of deducing Eq. (52), we could equally well introduce a complementary equation with the places of the nilpotents interchanged

$$
-\sigma^{\mu \nu} i \partial_{\mu} t_{\nu} \varphi_{\perp}=s_{-}(\gamma \cdot t)\left(\mathbf{1}+\gamma^{5}\right) i \partial_{\tau} \varphi_{\perp}+s_{+} \frac{M}{2}(\gamma \cdot t)\left(\mathbf{1}-\gamma^{5}\right) \varphi_{\perp}
$$

where we left the signs in place. Eq. (60) has all the features of Eq. (52), except that the role of the left and right handed spinors is interchanged. Writing the two relevant coupled equations analogous to Eqs. (56),(57) we get

$$
\begin{aligned}
& (\gamma \cdot t)(\sigma P t) \varphi_{\perp R}=s_{+} M \varphi_{\perp L} \\
& (\gamma \cdot t)(\sigma P t) \varphi_{\perp L}=2 s_{-} i \partial_{\tau} \varphi_{\perp R}
\end{aligned}
$$

Now, if we abide by Eq. (52), and describe the theory as portraying the evolution of a left-handed spinor, $\psi_{\perp L}$, while $\psi_{\perp R}$ is just a sort of auxiliary field, we give up righthanded events. This cannot be done without some justification. In Section we see that such an equation gives rise to a continuity equation, (through a simple procedure similar to the one performed for the Dirac equation), where the probability density is composed of left-handed spinors only.

The interpretation of $\psi_{\perp R}$ as an auxiliary field, justified by Eqs. (57), (56) where only $\psi_{\perp L}$ is seen to evolve according to $\tau$, the existence of the other equally justified equation (60), the inability to prefer Eq. (52) over Eq. (60), and the requirement for a reasonable probability density, lead us to a unification of the two.

We define

$$
\phi_{\perp} \equiv \psi_{\perp L}+\varphi_{\perp R} \quad \chi_{\perp} \equiv \psi_{\perp R}+\varphi_{\perp L}
$$

These are now four-spinors, where $\chi_{\perp}$ is an auxiliary field, and $\phi_{\perp}$ is the main field, evolving with $\tau$. The two sets of coupled equations (56),(57) and (51),(62), become

$$
\begin{aligned}
& (\gamma \cdot t)(\sigma P t) \phi_{\perp}=s_{+} M \chi_{\perp} \\
& (\gamma \cdot t)(\sigma P t) \chi_{\perp}=2 s_{-} i \partial_{\tau} \phi_{\perp}
\end{aligned}
$$

by just adding them up. We denote them as the "extended" form. We therefore have achieved a satisfactory probability density of four-spinors, which is sufficient for the two 
irreducible representations of $S L(2, C)$ (see [23, 32]). Of course by iteration we get the transverse Schrödinger-Stueckelberg equation for $\phi_{\perp}$ and for $\chi_{\perp}$.

\subsection{Longitudinal equations of motion}

In dealing with a first order equation that agrees with the longitudinal SchrödingerStueckelberg equation, Eq. (54), we check it for the nilpotents and momentum part we choose. Since the nilpotents are the same as for the transverse version all conclusions from Subsection apply here too. Iterating $L_{\|}(P)$ we get $P_{\|}^{2}=-(P \cdot t)^{2}$, and the relation between $s_{+}$to $s_{-}$is maintained as in the transverse case. The equation of motion is

$$
i\left(-i \partial_{\mu} t^{\mu}\right) \psi_{\|}=s_{-}(\gamma \cdot t)\left(\mathbf{1}-\gamma^{5}\right) i \partial_{\tau} \psi_{\|}+s_{+} \frac{M}{2}(\gamma \cdot t)\left(\mathbf{1}+\gamma^{5}\right) \psi_{\|}
$$

Using projectors of Eq. (55) we can decompose it in two

$$
\begin{aligned}
i(\gamma \cdot t)(P \cdot t) \psi_{\| L} & =s_{+} M \psi_{\| R} \\
i(\gamma \cdot t)(P \cdot t) \psi_{\| R} & =2 s_{-} i \partial_{\tau} \psi_{\| L}
\end{aligned}
$$

The discussion concerning both options in using the sign variables applies here as well,

and we do not repeat it. As for the transverse case, Eqs. (67),(68) can be used to show that the left and right handed spinors pertain to the longitudinal SchrödingerStueckelberg equation.

Exchanging the place of nilpotents in the equation to get the complementary equations of motion for the right handed part as the main field, while maintaining the interpretation of the auxiliary field, and adding the two types of equations as in the transverse case, leads to the coupled equations

$$
\begin{aligned}
& i(\gamma \cdot t)(P \cdot t) \phi_{\|}=s_{+} M \chi_{\|} \\
& i(\gamma \cdot t)(P \cdot t) \chi_{\|}=2 s_{-} i \partial_{\tau} \phi_{\|}
\end{aligned}
$$

with the same structure of $\phi_{\|}$and $\chi_{\|}$as in the transverse case.

\section{Probability density and currents}

The continuity equations for the transverse and longitudinal versions are obtained by using the procedure for obtaining the primary form of a continuity equation in Subsection 
. We show that the currents form consistent continuity equations, and are related to the Gordon decomposition of the Dirac current.

\subsection{Currents of the transverse equation}

Using the procedure in Subsection on Eq. (52), we obtain

$$
-\partial_{\mu}\left(\bar{\psi}_{\perp} \sigma^{\mu \nu} t_{\nu} \psi_{\perp}\right)=2 s_{-} \partial_{\tau}\left(\bar{\psi}_{\perp}(\gamma \cdot t) \frac{1}{2}\left(\mathbf{1}-\gamma^{5}\right) \psi_{\perp}\right)
$$

We observe that the R.H.S. of Eq. (71) is a probability density, exactly in the sense defined by Horwitz and Arshansky [23], but achieved without explicit group theoretical arguments. It is obviously positive definite for wave functions containing left handed components, which can be seen by transforming to a reference frame where $t_{\mu}=(1,0,0,0)$. We denote this probability density (in the chiral representation of $\gamma$ matrices) as

$$
\rho_{\perp L} \equiv\left(\bar{\psi}_{\perp}(\gamma \cdot t) \frac{1}{2}\left(\mathbf{1}-\gamma^{5}\right) \psi_{\perp}\right)=\psi_{\perp L}^{\dagger}\left(\tilde{\sigma}^{\mu} t_{\mu}\right) \psi_{\perp L}
$$

where

$$
\tilde{\sigma}^{\mu} \equiv\left(\mathbf{1},-\sigma^{i}\right) \quad ; \quad \sigma^{\mu} \equiv\left(\mathbf{1}, \sigma^{i}\right)
$$

and $\sigma^{i}$ are the Pauli matrices. The appearance of $\rho_{\perp L}$ only in Eq. (71) is the chief reason for introducing both Eqs. (52), (60). From Eq. (60) we obtain in the same way $\rho_{\perp R}$, and we can add them together, and obtain $\rho_{\perp}=\rho_{\perp L}+\rho_{\perp R}$, thus having the a density containing both $S L(2, C)$ representations as in [23, 32]. Since $\rho_{\perp R}$ is also positive definite, so is $\rho_{\perp}$, as required.

We define the four-currents in Eq. (71) as

$$
j_{\perp L}^{\mu} \equiv \bar{\psi}_{\perp}\left(\sigma^{\mu \nu} t_{\nu}\right) \psi_{\perp}
$$

so we actually have

$$
-\partial_{\mu} j_{\perp L}^{\mu}=2 s_{-} \partial_{\tau} \rho_{\perp L}
$$

which is the five dimensional conservation theorem required. Let us consider

$$
\bar{\psi}_{\perp L}(\sigma P t) \psi_{\perp R}=2 s_{-} \bar{\psi}_{\perp L}(\gamma \cdot t) i \partial_{\tau} \psi_{\perp L}
$$


and the conjugate of Eq. (76)

$$
\bar{\psi}_{\perp R}(\sigma \overleftarrow{P} t) \psi_{\perp L}=2 s_{-}\left(i \partial_{\tau} \bar{\psi}_{\perp L}\right)(\gamma \cdot t) \psi_{\perp L}
$$

Using the equations of motion, Eqs. (56), (57), we find

$$
\begin{aligned}
\partial_{\tau} \psi_{\perp R} & =\frac{1}{s_{+} M}(\gamma \cdot t)(\sigma P t)\left(\partial_{\tau} \psi_{\perp L}\right) \\
\partial_{\tau} \bar{\psi}_{\perp R} & =\frac{1}{s_{+} M}\left(\partial_{\tau} \bar{\psi}_{\perp L}\right)(\gamma \cdot t)(\sigma \overleftarrow{P} t) \\
\partial_{\tau} \psi_{\perp L} & =-\frac{i}{2 s_{-}}(\gamma \cdot t)(\sigma P t) \psi_{\perp R} \\
\partial_{\tau} \bar{\psi}_{\perp L} & =\frac{i}{2 s_{-}} \bar{\psi}_{\perp R}(\gamma \cdot t)(\sigma \overleftarrow{P} t)
\end{aligned}
$$

Using Eq. (56), and the sum of Eqs. (76), (77) we get

$$
\begin{aligned}
\partial_{\tau} \rho_{\perp L} & =-\frac{i}{2 M} \bar{\psi}_{\perp L}((\sigma P t)(\gamma \cdot t)(\sigma P t)+(\gamma \cdot t)(\sigma \overleftarrow{P} t)(\sigma \overleftarrow{P} t)) \psi_{\perp L} \\
& =\frac{i}{2 M} \bar{\psi}_{\perp L}(\gamma \cdot t)\left(-\vec{P}_{\perp}^{2}+\overleftarrow{P_{\perp}^{2}}\right) \psi_{\perp L}
\end{aligned}
$$

To simplify the expression for $\rho_{\perp L}$ we state some useful relations

$$
\begin{aligned}
P_{\perp \mu} & \equiv-i \partial_{\perp \mu} \equiv-i \partial_{\mu}+\left(-i \partial_{\nu} \cdot t^{\nu}\right) t_{\mu} \\
P_{\perp}^{2} & =-\partial_{\perp \mu} \partial_{\perp}^{\mu}
\end{aligned}
$$

In the reference frame where $t_{\mu}=(1,0,0,0)$ the transverse derivative is seen to be

$$
\begin{array}{r}
\partial_{\perp 0}=0 \\
\partial_{\perp i}=\partial_{i}
\end{array}
$$

So,

$$
\partial_{\tau} \rho_{\perp L}=\frac{i}{2 M} \partial_{\perp \mu}\left(\bar{\psi}_{\perp L}(\gamma \cdot t) \overleftrightarrow{\partial_{\perp}^{\mu}} \psi_{\perp L}\right)
$$

Using Eqs. (78) we find that the $\tau$ derivative of the auxiliary field also obeys a continuity equation, exactly as the main field with $\psi_{\perp L}$ exchanged with $\psi_{\perp R}$

$$
\partial_{\tau} \rho_{\perp L_{a u x}}=\frac{i}{2 M} \partial_{\perp \mu}\left(\bar{\psi}_{\perp R}(\gamma \cdot t) \overleftrightarrow{\partial_{\perp}^{\mu}} \psi_{\perp R}\right)
$$


where we therefore understand $\rho_{\perp L_{a u x}}$ as a "density" associated with the auxiliary field of $\psi_{\perp L}$, namely with $\psi_{\perp R}$. This form of current resembles the Klein-Gordon current, and when taken in a frame where $t_{\mu}=(1,0,0,0)$ we recover the space part of the current.

To make a connection with the form of currents exhibited by the Dirac equation, we re-express Eq. (71) as

$$
-t_{\nu} \tilde{j}_{\perp L}^{\nu}=2 s_{-} t_{\mu} \partial_{\tau} \tilde{\rho}_{\perp L}^{\mu}
$$

where

$$
\begin{aligned}
& \tilde{j}_{\perp L}^{\nu} \equiv \partial_{\mu}\left(\bar{\psi}_{\perp} \sigma^{\mu \nu} \psi_{\perp}\right) \\
& \tilde{\rho}_{\perp L}^{\mu} \equiv \bar{\psi}_{\perp L} \gamma^{\mu} \psi_{\perp L}
\end{aligned}
$$

Now we integrate over a space-like hypersurface enclosing a four-dimensional volume, where $t_{\mu}$ is normal to the surface (see, for example, [37]). Therefore, we imagine a space-like hypersurface perpendicular to $t_{\mu}$ and another one like it some distance above, meeting it only at infinity; it then follows from Eq. (84) that

$$
-\int_{\Sigma_{t}} d \Sigma t_{\nu} \tilde{j}_{\perp L}^{\nu}=2 s_{-} \partial_{\tau} \int_{\Sigma_{t}} d \Sigma t_{\mu} \tilde{\rho}_{\perp L}^{\mu}
$$

where $\Sigma_{t}$ denotes the closed space-like hypersurface. We transform to the four-divergence

$$
-\int_{V_{t}} d v \partial_{\nu} \tilde{j}_{\perp L}^{\nu}=2 s_{-} \partial_{\tau} \int_{V_{t}} d v \partial_{\mu} \tilde{\rho}_{\perp L}^{\mu}
$$

But since

$$
-\partial_{\nu} \tilde{j}_{\perp L}^{\nu}=\partial_{\nu} \partial_{\mu}\left(\bar{\psi}_{\perp} \sigma^{\mu \nu} \psi_{\perp}\right)=0
$$

on account of the antisymmetry of $\sigma^{\mu \nu}$ and the symmetry of $\partial_{\mu} \partial_{\nu}$, we get

$$
\int_{V_{t}} d v \partial_{\tau} \partial_{\mu} \tilde{\rho}_{\perp L}^{\mu}=0
$$

Now, assuming this is true for the integrand also, i.e., for any thin slice $V_{t}$, we obtain

$$
\partial_{\tau} \partial_{\mu}\left(\bar{\psi}_{\perp L} \gamma^{\mu} \psi_{\perp L}\right)=0
$$

and hence

$$
\partial_{\mu}\left(\bar{\psi}_{\perp L} \gamma^{\mu} \psi_{\perp L}\right)=f\left(x^{\mu}\right)
$$

The quantity $\partial_{\mu}\left(\bar{\psi}_{\perp L} \gamma^{\mu} \psi_{\perp L}\right)$ is independent of $\tau$, and if it is not zero, it is some $\tau$ independent function $f\left(x^{\mu}\right)$. 
Comparing to the Gordon decomposition of the Dirac currents one finds a similar structure when applying the continuity equation.

We now consider the combination of the above methods and equations concerning Eq. (52), with their application to the nilpotent interchanged equation, Eq. (50). The same line of thought, when applied to Eq. (60), yields the same results, only $\varphi_{\perp R}$ replaces $\psi_{\perp L}, \varphi_{\perp L}$ replaces $\psi_{\perp R}, \partial_{\tau} \rho_{\perp R}$ replaces $\partial_{\tau} \rho_{\perp L}$. Ultimately we get the continuity equation for the main field

$$
\partial_{\tau}\left(\bar{\phi}_{\perp}(\gamma \cdot t) \phi_{\perp}\right)=\frac{i}{2 M} \partial_{\perp \mu}\left(\bar{\phi}_{\perp}(\gamma \cdot t) \overleftrightarrow{\partial_{\perp}^{\mu}} \phi_{\perp}\right)
$$

Furthermore, one obtains for the total main field, a $\tau$ independent continuity equation resembling the one in Dirac's theory

$$
\partial_{\tau} \partial_{\mu}\left(\bar{\phi}_{\perp} \gamma^{\mu} \phi_{\perp}\right)=0
$$

If $\partial_{\mu}\left(\bar{\phi}_{\perp} \gamma^{\mu} \phi_{\perp}\right)=0$, (i.e., in case the sum of divergences for $\psi_{\perp L}$ and $\varphi_{\perp R}$ cancel), we have the form of the Dirac continuity equation, but for the transverse field (recall that the wave function $\phi_{\perp}$ itself still has dependence on $t_{\mu}$ ).

We have a physical behavior of the particles described by our equations, which resembles the physical behavior of Dirac's particles, without the use of Dirac's equation, but using instead the transverse equation. It is interesting to observe that the Dirac type currents were obtained from the probability density of our theory. We may view this as though our theory is in some sense a fundamental underlying structure for what is essentially Dirac's theory.

We remark, moreover, that we have obtained in our method, a scalar product which agrees with that of [23], i.e.,

$$
\left(\phi_{\perp 1}, \phi_{\perp 2}\right)=\int d^{4} x \bar{\phi}_{\perp 1}(\gamma \cdot t) \phi_{\perp 2}
$$

\subsection{Currents of the longitudinal equation}

Using the procedure in Subsection on Eq. (66), we obtain

$$
\bar{\psi}_{\|} \stackrel{\leftrightarrow}{\partial}_{\mu} t^{\nu} \psi_{\|}=2 s_{-} i \partial_{\tau}\left(\bar{\psi}_{\|}(\gamma \cdot t) \frac{1}{2}\left(\mathbf{1}-\gamma^{5}\right) \psi_{\|}\right)
$$

In the R.H.S. we identify a positive definite quantity. Unfortunately, we cannot understand Eq. (96) as a continuity equation. However, we can overcome the problem by 
observing similar features of the equations as in the transverse case. We state some features of the longitudinal momentum

$$
\begin{aligned}
P_{\| \mu} & \equiv-i \partial_{\| \mu} \equiv-\left(-i \partial_{\nu} t^{\nu}\right) t_{\mu} \\
P_{\|}^{2} & =-\partial_{\| \mu} \partial_{\|}^{\mu}
\end{aligned}
$$

and in the special frame where $t_{\mu}=(1,0,0,0)$ we have

$$
\begin{aligned}
& \partial_{\| 0}=\partial_{0} \\
& \partial_{\| i}=0
\end{aligned}
$$

For a treatment analogous to that of the transverse equation we find

$$
\partial_{\tau} \rho_{\| L}=\frac{i}{2 M} \partial_{\| \mu}\left(\bar{\psi}_{\| L}(\gamma \cdot t) \overleftrightarrow{\partial_{\|}^{\mu}} \psi_{\| L}\right)
$$

Using the space-like hypersurface integration while defining

$$
\begin{aligned}
\tilde{j}_{\| L}^{\nu} & \equiv \bar{\psi}_{\|} \stackrel{\partial^{\mu}}{ } \psi_{\|} \\
\tilde{\rho}_{\| L}^{\mu} & \equiv \bar{\psi}_{\| L} \gamma^{\mu} \psi_{\| L} \\
t_{\nu} \tilde{j}_{\| L}^{\nu} & =2 s_{-} t_{\mu} i \partial_{\tau} \tilde{\rho}_{\| L}^{\mu}
\end{aligned}
$$

we get

$$
\int_{V_{t}} d v 2 s_{-} \partial_{\tau} \partial_{\mu}\left(\bar{\psi}_{\| L} \gamma^{\mu} \psi_{\| L}\right)=-\int_{V_{t}} d v \partial_{\nu}\left(\bar{\psi}_{\|} i \stackrel{\leftrightarrow}{\partial^{\nu}} \psi_{\|}\right)
$$

Eq. (101) is similar to Eq. (88) in the sense that the probability density gives rise to a form of a Dirac current. The transverse current of Eq. (88) has the form of the spin current of the Gordon decomposition of the Dirac current, while the longitudinal current of Eq. (101) has the form of the convection part. It seems that the two parts of the Gordon decomposition manifest themselves in the two versions of the equations of motion. In case $\partial_{\mu}\left(\bar{\psi}_{\| L} \gamma^{\mu} \psi_{\| L}\right)$ is independent of $\tau$, one would have a result for $\partial_{\nu}\left(\bar{\psi}_{\|} i \overleftrightarrow{\partial^{\nu}} \psi_{\|}\right)$, similar to that of the transverse part. If we integrate Eq. (101) over $\tau$, one obtains a conservation law of the same type as well.

Finally we state the equation for $\phi_{\|}$, the four-spinor main field

$$
\partial_{\tau} \rho_{\|}=\frac{i}{2 M} \partial_{\| \mu}\left(\bar{\phi}_{\|}(\gamma \cdot t) \overleftrightarrow{\partial_{\|}^{\mu}} \phi_{\|}\right)
$$

The solution of the equations of motion is given in Appendix B. 


\section{Product Hilbert space}

The vector $t_{\mu}$ splits the evolution of the momentum into two parts, the transverse and the longitudinal. These two modes of the motion of an event, transverse and longitudinal, are complementary. Therefore, the overall Hilbert space is the tensor product of the transverse and longitudinal Hilbert spaces

$$
\mathcal{H}=\mathcal{H}_{\perp} \otimes \mathcal{H}_{\|}
$$

Considering the free event, the probability density is

$$
\rho=\rho_{\perp} \cdot \rho_{\|}
$$

By defining

$$
\begin{aligned}
L_{\perp} & =(\gamma \cdot t)(\sigma P t) \\
L_{\|} & =i(\gamma \cdot t)(P \cdot t) \\
\omega & =2 M
\end{aligned}
$$

and absorbing the signs $s_{+}, s_{-}$into $\phi$ or $\chi$, the transverse and longitudinal equations can be expressed in a similar manner

$$
\begin{aligned}
& L_{\perp} \phi_{\perp}=\omega \chi_{\perp} \\
& L_{\perp} \chi_{\perp}=i \partial_{\tau} \phi_{\perp}
\end{aligned}
$$

and

$$
\begin{aligned}
L_{\|} \phi_{\|} & =\omega \chi_{\|} \\
L_{\|} \chi_{\|} & =i \partial_{\tau} \phi_{\|}
\end{aligned}
$$

In the product Hilbert space we define

$$
\begin{aligned}
\phi & =\phi_{\perp} \otimes \phi_{\|} \\
\chi & =\chi_{\perp} \otimes \chi_{\|}
\end{aligned}
$$

and $L_{\perp}, L_{\|}$operate on their respective factors. Now

$$
\begin{aligned}
L_{\perp} \phi & =\omega\left(\chi_{\perp} \otimes \phi_{\|}\right) \\
L_{\|} \phi & =\omega\left(\phi_{\perp} \otimes \chi_{\|}\right) \\
L_{\perp} \chi & \left.=\left(i \partial_{\tau} \phi_{\perp}\right) \otimes \chi_{\|}\right) \\
L_{\|} \chi & =\chi_{\perp} \otimes\left(i \partial_{\tau} \phi_{\|}\right)
\end{aligned}
$$


Denoting

$$
L=L_{\perp} \otimes L_{\|}
$$

we obtain

$$
\begin{aligned}
L \phi & =\omega^{2} \chi \\
L \chi & =\left(i \partial_{\tau} \phi_{\perp}\right) \otimes\left(i \partial_{\tau} \phi_{\|}\right)
\end{aligned}
$$

and

$$
L^{2} \phi=L^{2} \chi=\left(\omega i \partial_{\tau} \phi_{\perp}\right) \otimes\left(\omega i \partial_{\tau} \phi_{\|}\right)
$$

We are interested in getting a unified equation for the transverse and longitudinal modes, conforming to the full Schrödinger-Stueckelberg equation. Therefore we consider the combinations

$$
\begin{aligned}
\left(L_{\perp}+L_{\|}\right) \phi & =\omega\left(\chi_{\perp} \otimes \phi_{\|}+\phi_{\perp} \otimes \chi_{\|}\right) \\
\left(L_{\perp}+L_{\|}\right) \chi & =\left(i \partial_{\tau} \phi_{\perp}\right) \otimes \chi_{\|}+\chi_{\perp} \otimes\left(i \partial_{\tau} \phi_{\|}\right)
\end{aligned}
$$

Considering $\left(L_{\perp}+L_{\|}\right)^{2}$ we find

$$
\begin{aligned}
\left(L_{\perp}+L_{\|}\right)^{2} \phi & =\omega i \partial_{\tau} \phi+2 \omega^{2} \chi \\
& =\omega i \partial_{\tau} \phi+2 L \phi
\end{aligned}
$$

Since

$$
\left(L_{\perp}+L_{\|}\right)^{2}=L_{\perp}^{2}+L_{\|}^{2}+2 L
$$

we obtain

$$
\left(L_{\perp}^{2}+L_{\|}^{2}\right) \phi=\omega i \partial_{\tau} \phi
$$

which is the desired full Schrödinger-Stueckelberg equation. Repeating the same steps for the auxiliary field $\chi$, we find that it also obeys the full Schrödinger-Stueckelberg equation

$$
\left(L_{\perp}^{2}+L_{\|}^{2}\right) \chi=\omega i \partial_{\tau} \chi
$$




\section{Interacting charged equations}

To introduce the electromagnetic coupling, we define the electromagnetic field appropriately on the manifold of the tensor product space i.e.,

$$
a^{\alpha}(x, \tau) \equiv a^{\alpha}\left(x_{\perp}, x_{\|}, \tau\right)
$$

where $\alpha=0,1,2,3, \tau$. We may therefore define

$$
\begin{aligned}
L_{\perp}^{\Pi} & =(\gamma \cdot t)(\sigma \Pi t) \\
L_{\|}^{\Pi} & =i(\gamma \cdot t)(\Pi \cdot t)
\end{aligned}
$$

where

$$
\Pi^{\mu}=P^{\mu}-e a^{\mu}
$$

Note that although $L_{\perp}$ and $L_{\|}$act on the tensor product space as $L_{\perp} \otimes \mathbf{1}$ and $\mathbf{1} \otimes L_{\|}$, this is no longer true, in general, for $L_{\perp}^{\Pi}$ and $L_{\|}^{\Pi}$. Let us start by defining equations containing fields of the form $a_{\perp \mu}$ and $a_{\| \mu}$

$$
\begin{gathered}
(\gamma \cdot t)\left[\sigma^{\mu \nu}\left(P_{\mu}-e a_{\perp \mu}\left(x_{\perp}, \tau\right)\right) t_{\nu}\right]\left(\phi_{\perp}\left(x_{\perp}, \tau\right) \otimes \mathbf{1}\right)=\omega\left(\chi_{\perp}\left(x_{\perp}, \tau\right) \otimes \mathbf{1}\right) \\
(\gamma \cdot t)\left[\sigma^{\mu \nu}\left(P_{\mu}-e a_{\perp \mu}\left(x_{\perp}, \tau\right)\right) t_{\nu}\right]\left(\chi_{\perp}\left(x_{\perp}, \tau\right) \otimes \mathbf{1}\right)=\left[\left(i \partial_{\tau}+e a_{\perp \tau}\left(x_{\perp}, \tau\right)\right) \phi_{\perp}\left(x_{\perp}, \tau\right)\right] \otimes \mathbf{1}
\end{gathered}
$$

and

$$
\begin{gathered}
i(\gamma \cdot t)\left[\left(P_{\mu}-e a_{\| \mu}\left(x_{\|}, \tau\right)\right) t^{\mu}\right]\left(\mathbf{1} \otimes \phi_{\|}\left(x_{\|}, \tau\right)\right)=\omega\left(\mathbf{1} \otimes \chi_{\|}\left(x_{\perp}, \tau\right)\right) \\
i(\gamma \cdot t)\left[\left(P_{\mu}-e a_{\| \mu}\left(x_{\|}, \tau\right)\right) t^{\mu}\right]\left(\mathbf{1} \otimes \chi_{\|}\left(x_{\|}, \tau\right)\right)=\mathbf{1} \otimes\left[\left(i \partial_{\tau}+e a \| \tau\left(x_{\|}, \tau\right)\right) \phi_{\|}\left(x_{\|}, \tau\right)\right]
\end{gathered}
$$

Now, since we can write

$$
\sum\left(a_{\perp}^{n} \phi_{\perp} \otimes a_{\|}^{n} \phi_{\|}\right)\left(x_{\perp}, x_{\|}\right)=\sum a_{\perp}^{n}\left(x_{\perp}\right) a_{\|}^{n}\left(x_{\|}\right) \phi_{\perp}\left(x_{\perp}\right) \phi_{\|}\left(x_{\|}\right)
$$

where $n$ is some index, the limit of this sum can approximate any function $a(x)$ i.e.,

$$
\sum a_{\perp}^{n}\left(x_{\perp}\right) a_{\|}^{n}\left(x_{\|}\right) \phi_{\perp}\left(x_{\perp}\right) \phi_{\|}\left(x_{\|}\right)=a(x) \phi_{\perp}\left(x_{\perp}\right) \phi_{\|}\left(x_{\|}\right)
$$

Since we have gauge invariance

$$
\begin{gathered}
a_{\perp \mu}\left(x_{\perp}\right) \rightarrow a_{\perp \mu}\left(x_{\perp}\right)+\frac{1}{e} \partial_{\perp \mu} \Lambda_{\perp}\left(x_{\perp}\right) \\
a_{\| \mu}\left(x_{\|}\right) \rightarrow a_{\| \mu}\left(x_{\|}\right)+\frac{1}{e} \partial_{\| \mu} \Lambda_{\|}\left(x_{\|}\right)
\end{gathered}
$$


we may generalize the gauge invariance to

$$
a_{\mu}\left(x_{\perp}, x_{\|}, \tau\right) \rightarrow a_{\mu}\left(x_{\perp}, x_{\|}, \tau\right)+\frac{1}{e} \partial_{\mu} \Lambda\left(x_{\perp}, x_{\|}, \tau\right)
$$

and

$$
a_{\tau}\left(x_{\perp}, x_{\|}, \tau\right) \rightarrow a_{\tau}\left(x_{\perp}, x_{\|}, \tau\right)-\frac{1}{e} \partial_{\tau} \Lambda\left(x_{\perp}, x_{\|}, \tau\right)
$$

These hold for the equations

$$
\begin{aligned}
(\gamma \cdot t)(\sigma \Pi t)_{\perp}\left(\phi_{\perp} \otimes \phi_{\|}\right) & =\omega\left(\chi_{\perp} \otimes \phi_{\|}\right) \\
(\gamma \cdot t)(\sigma \Pi t)_{\perp}\left(\chi_{\perp} \otimes \chi_{\|}\right) & =\left(i \partial_{\tau} \phi_{\perp}\right) \otimes \chi_{\|}+e a_{\tau}\left(\phi_{\perp} \otimes \chi_{\|}\right) \\
i(\gamma \cdot t)(\Pi \cdot t)_{\|}\left(\phi_{\perp} \otimes \phi_{\|}\right) & =\omega\left(\phi_{\perp} \otimes \chi_{\|}\right) \\
i(\gamma \cdot t)(\Pi \cdot t)_{\|}\left(\chi_{\perp} \otimes \chi_{\|}\right) & =\chi_{\perp} \otimes\left(i \partial_{\tau} \phi_{\|}\right)+e a_{\tau}\left(\chi_{\perp} \otimes \phi_{\|}\right)
\end{aligned}
$$

since in each equation we can refer to the other variable (say $\|$ in the $\perp$ equation), as a parameter. The subscript on the matrix operators on the L.H.S. of the equations means that we operate with derivatives on the relevant factor space.

We can therefore define the form of the basic equations as

$$
\begin{aligned}
L_{\perp}^{\Pi} \phi_{\perp} & =\omega \chi_{\perp} \\
L_{\perp}^{\Pi} \chi_{\perp} & =\left(i \partial_{\tau}+e a_{\tau}\right) \phi_{\perp}
\end{aligned}
$$

and

$$
\begin{aligned}
L_{\|}^{\Pi} \phi_{\|} & =\omega \chi_{\|} \\
L_{\|}^{\Pi} \chi_{\|} & =\left(i \partial_{\tau}+e a_{\tau}\right) \phi_{\|}
\end{aligned}
$$

Denoting $L^{\Pi}$ as the tensor product operator obtained from $L_{\perp}^{\Pi}$ and $L_{\|}^{\Pi}$, as

$$
L^{\Pi}=L_{\perp}^{\Pi} \otimes L_{\|}^{\Pi}
$$

we obtain

$$
\begin{aligned}
L^{\Pi} \phi & =\omega^{2} \chi \\
L^{\Pi} \chi & =\left(i \partial_{\tau} \phi_{\perp}\right) \otimes\left(i \partial_{\tau} \phi_{\|}\right)+e^{2} a_{\tau}^{2} \phi+e a_{\tau} i \partial_{\tau} \phi
\end{aligned}
$$


As before, we obtain from the squared expression

$$
\begin{aligned}
\left(L_{\perp}^{\Pi}+L_{\|}^{\Pi}\right)^{2} \phi & =\omega\left(i \partial_{\tau}+e a_{\tau}\right) \phi+2 \omega^{2} \chi \\
& =\omega\left(i \partial_{\tau}+e a_{\tau}\right) \phi+2 L^{\Pi} \phi
\end{aligned}
$$

Therefore we find

$$
\left[\left(L_{\perp}^{\Pi}\right)^{2}+\left(L_{\|}^{\Pi}\right)^{2}\right] \phi=\omega\left(i \partial_{\tau}+e a_{\tau}\right) \phi
$$

which is exactly the full electromagnetically coupled Schrödinger-Stueckelberg equation. Now, using the fact that

$$
\left(\sigma^{\mu \nu} \Pi_{\mu} t_{\nu}\right)\left(\sigma^{\rho \lambda} \Pi_{\rho} t_{\lambda}\right)=\frac{1}{4}\left(\left[\Pi_{\mu}, \Pi_{\rho}\right]\left[\sigma^{\mu \nu} t_{\nu}, \sigma^{\mu \rho} t_{\lambda}\right]+\left\{\Pi_{\mu}, \Pi_{\rho}\right\}\left\{\sigma^{\mu \nu} t_{\nu}, \sigma^{\mu \rho} t_{\lambda}\right\}\right)
$$

the definition (this quantity was defined by Horwitz and Arshansky [23] as well)

$$
-2 i \sigma_{t}^{\mu \rho}=\left[\sigma^{\mu \nu} t_{\nu}, \sigma^{\rho \lambda} t_{\lambda}\right]=2 i\left(\sigma^{\mu \rho} t^{2}-\left(\sigma^{\mu \lambda} t_{\lambda}\right) t^{\rho}-\left(\sigma^{\nu \rho} t_{\nu}\right) t^{\mu}\right)
$$

and

$$
\left\{\sigma^{\mu \nu} v_{\nu}, \sigma^{\rho \lambda} v_{\lambda}\right\}=2\left(v^{2} g^{\mu \rho}-v^{\mu} v^{\rho}\right)
$$

and the relation

$$
\left[\Pi_{\mu}, \Pi_{\nu}\right]=i e f_{\mu \nu}
$$

we get

$$
\left(L_{\perp}^{\Pi}\right)^{2}=\Pi_{\perp}^{2}-\frac{e}{2} \sigma_{t}^{\mu \nu} f_{\mu \nu}
$$

by defining

$$
\Pi_{\perp \mu} \equiv \Pi_{\mu}+(\Pi \cdot t) t_{\mu}
$$

Furthermore, we have

$$
\begin{aligned}
\left(L_{\|}^{\Pi}\right)^{2} & =-(\Pi \cdot t)^{2} \\
\Pi_{\perp}^{2} & =\Pi^{2}+(\Pi \cdot t)^{2}
\end{aligned}
$$

and it is easy to see that Eq. (155) is

$$
\left(i \partial_{\tau}+e a_{\tau}\right) \phi=\frac{1}{2 M}\left[\Pi^{2}-\frac{e}{2} \sigma_{t}^{\mu \nu} f_{\mu \nu}\right] \phi
$$


which is exactly the same equation found by Horwitz and Arshansky [23]. This shows that our formulation is fully consistent with theirs.

The gyromagnetic ratio is taken as the ratio between the coefficients of the terms $\Pi^{2}$ and $\frac{e}{2} \sigma_{t}^{\mu \nu} f_{\mu \nu}$, giving the correct relation of relative size and sign, between the momentum, spin coupling, and mass terms (see 34]). Furthermore, we obtain, as in Horwitz and Arshansky [23, a fully Hermitian spin term, as opposed to the Dirac case (and the additional $a_{\tau}$ field which they did not use).

\section{The Dirac limit}

We have seen that it is impossible under the assumptions of Section to find a single first order equation which iterates to the full Schrödinger-Stueckelberg equation. Therefore, we resorted to the combined product Hilbert space description in Section . However, by making a slight modification we can obtain the full Schrödinger-Stueckelberg equation by iteration from a single equation, but there is a price to pay; the gyromagnetic term in the electromagnetically coupled version is no longer Hermitian, like in Dirac's three dimensional case, and the structure of the continuity equation is lost. This modification is the necessary ingredient to see the connection between the theory developed so far and Dirac's theory.

The modified approach that we use to employ a single equation is to break up the operator $L(P)$ to two parts, which have different algebraic structure with respect to the nilpotents $N_{ \pm}$. This will enable us to obtain the full Schrödinger-Stueckelberg equation upon iteration.

Let us assume that $L(P)$ is composed explicitly of two parts, $L_{1}(P)$ and $L_{2}(P)$. Then, the general form of the first order equation is

$$
\left(L_{1}(P)+L_{2}(P)\right) \psi=s_{-} N_{-} i \partial_{\tau} \psi+s_{+} M N_{+} \psi
$$

We now multiply Eq. (163) from the left by $\left(L_{1}(P)-L_{2}(P)\right)$. To be able to insert Eq. (163) into the new equation we must have

$$
\begin{array}{r}
\left\{L_{1}(P), N_{ \pm}\right\}=0 \\
{\left[L_{2}(P), N_{ \pm}\right]=0}
\end{array}
$$


Requiring that

$$
\left[L_{1}(P), L_{2}(P)\right]=0
$$

and that $N_{ \pm}$are nilpotents, we obtain

$$
\left(L_{1}^{2}(P)-L_{2}^{2}(P)\right) \psi=-s_{-} s_{+} M \tilde{C}_{n n} i \partial_{\tau} \psi
$$

Performing an evaluation process similar to the one done in Section, we make the simplest choice which is

$$
L_{1}(P) \equiv(\sigma P t) \quad ; \quad L_{2}(P) \equiv i(P \cdot t)
$$

and the nilpotents are $N_{ \pm}=(\gamma \cdot t)\left(\mathbf{1} \pm \gamma^{5}\right)$. This is a synthesis of the use of commutators and anticommutators of Section, and of the transverse and longitudinal equations. The equation of motion is

$$
\left(-\sigma^{\mu \nu} i \partial_{\mu} t_{\nu}+i\left(-i \partial_{\mu} t^{\mu}\right)\right) \psi=s_{-}(\gamma \cdot t)\left(\mathbf{1}-\gamma^{5}\right) i \partial_{\tau} \psi+s_{+} \frac{M}{2}(\gamma \cdot t)\left(\mathbf{1}+\gamma^{5}\right) \psi
$$

Iterating we get the full Schrödinger-Stueckelberg equation (1). We obtain the currents

$$
\left.-i \partial_{\nu}\left(\bar{\psi} \sigma^{\nu \mu} t_{\mu} \psi\right)+\bar{\psi} \overleftrightarrow{\partial}^{\mu} t_{\mu} \psi\right)=2 s_{-} i \partial_{\tau}\left(\bar{\psi}_{L}(\gamma \cdot t) \psi_{L}\right)
$$

which can be written as

$$
-t_{\mu} j_{L}^{\mu}=2 \partial_{\tau} s_{-} t_{\mu}\left(\bar{\psi}_{L} \gamma^{\mu} \psi_{L}\right)
$$

where

$$
j_{L}^{\mu} \equiv\left(\partial_{\nu}\left(\bar{\psi} \sigma^{\nu \mu} \psi\right)+\bar{\psi} i \stackrel{\leftrightarrow}{\partial^{\mu}} \psi\right)
$$

It is clearly seen that $j_{L}^{\mu}$ resembles the definition of the Gordon decomposition of currents of the Dirac equation.

An equation with the roles of $N_{ \pm}$interchanged can built, resulting in a similar Gordon decomposition but this time only right-right terms appear in the current. When we discussed the transverse and longitudinal equations we could transform between the two versions opposite in the nilpotent assignment by a suitable transformation. Decomposing Eq. (168) by projection into two coupled equations we find

$$
\begin{aligned}
-i(\gamma \cdot P) \psi_{L} & =s_{+} M \psi_{R} \\
-i(\gamma \cdot P) \psi_{R} & =2 s_{-} i \partial_{\tau} \psi_{L}
\end{aligned}
$$


At this stage the $t_{\mu}$ dependence of the equations disappears; we deal with this below. Taking these equations to mass shell, choosing $s_{+}=s_{-}=-1$, and observing the eigenvalues of plane wave solutions (see Appendix B), we obtain

$$
\begin{aligned}
-i(\gamma \cdot P) \psi_{L} & =-m \psi_{R} \\
-i(\gamma \cdot P) \psi_{R} & =m \psi_{L}
\end{aligned}
$$

and for the equation with $N_{ \pm}$interchanged

$$
\begin{aligned}
-i(\gamma \cdot P) \varphi_{R} & =-m \varphi_{L} \\
-i(\gamma \cdot P) \varphi_{L} & =m \varphi_{R}
\end{aligned}
$$

To transform from Eq. (173) to Eq. (174), we can multiply $\psi$ by $-\frac{1}{m}(\gamma \cdot P)$ (working on shell) to exchange the roles of the left and right handed spinors, therefore the relation between $\psi$ and $\varphi$ is

$$
-\frac{\sigma^{\mu} p_{\mu}}{m} \psi_{R}=\varphi_{L} \quad ; \quad-\frac{\tilde{\sigma}^{\mu} p_{\mu}}{m} \psi_{L}=\varphi_{R}
$$

This implies that

$$
\varphi_{L}=-i \psi_{L} \quad ; \quad \varphi_{R}=i \psi_{R}
$$

and the relation between the main field $\phi$ and the auxiliary field $\chi$ is

$$
\phi=i \chi
$$

Using Eq. (176), Eqs. (173),(174) are then exactly the Dirac equation in the chiral representation (holding for both the main and auxiliary fields)

$$
\begin{aligned}
(\gamma \cdot P) \phi_{R} & =-m \phi_{L} \\
(\gamma \cdot P) \phi_{L} & =-m \phi_{R}
\end{aligned}
$$

This implies, of course, the Dirac current and continuity equations for the main and auxiliary fields $\phi$ and $\chi$.

When electromagnetic coupling is introduced in Eq. (168), assuming that $a_{\tau}=0$ (Coulomb-like gauge) and $a_{\mu}$ independent of $\tau$ (restricting ourselves to the zero mode) for simplicity, we obtain for the second order equation

$$
i \partial_{\tau} \psi=\frac{1}{2 M}\left[\Pi^{2}-\frac{e}{2} \sigma^{\mu \nu} f_{\mu \nu}\right] \psi
$$


which has the usual non-hermitian term, and conforms to the second order charged Dirac equation (when $i \partial_{\tau} \rightarrow-\frac{m^{2}}{2 M}$, see [7]). We therefore see that the decomposition into transverse and longitudinal modes was essential to achieve a Hermitian interaction as well as the Schrödinger-Stueckelberg form.

All the discussion above can be approached from a different point of view. If we take the longitudinal equations of motion, and assume that the momentum is in the direction of the four-vector $t_{\mu}$, such that

$$
P_{\mu}=\alpha t_{\mu} \quad ; \quad P^{2}=-\alpha^{2}
$$

then the longitudinal equations of motion become

$$
\begin{aligned}
-i(\gamma \cdot P) \psi_{\| L} & =s_{+} M \psi_{\| R} \\
-i(\gamma \cdot P) \psi_{\| R} & =2 s_{-} i \partial_{\tau} \psi_{\| L}
\end{aligned}
$$

with a similar result for the nilpotent interchanged equation. Making the identification $\psi=\psi_{\|}$, these are exactly Eqs. (172). One can now understand how $t_{\mu}$ disappeared previously in Eqs. (172). Furthermore, looking at the basic structure of the solutions of the longitudinal equation for $\phi_{\|}$(see Appendix B), Eq. (244), and replacing $t_{\mu}$ by $\frac{p_{\mu}}{m}$ in $\zeta_{\|}^{-}(t)$, (on shell condition), we obtain the exact non-normalized solutions of the Dirac equation in the chiral representation

$$
\zeta_{1}(p) \equiv\left(\begin{array}{c}
-\frac{1}{m} \sigma^{\mu} p_{\mu} \xi_{1} \\
\xi_{1}
\end{array}\right) \quad \zeta_{2}(p) \equiv\left(\begin{array}{c}
\xi_{2} \\
-\frac{1}{m} \tilde{\sigma}^{\mu} p_{\mu} \xi_{2}
\end{array}\right)
$$

where $\xi_{1,2}$ are two independent two-spinors; this is also apparent from Eq. (175) (actually, when taken in the energy representation of the Dirac matrices these solutions correspond to the positive energy, and $\zeta_{\|}^{+}(t)$ solves the Dirac equation for the negative energy). The transformation of the extended helicity projection operator (see Appendix B) is a bit delicate since $(\sigma P t)$ and $\left|p_{\perp}\right|$ go to zero together. Observing Eq. (168) and its consequent Eqs. (172), we find the extended helicity operator commutes with them. In Eqs. (172) $t_{\mu}$ does not appear, so we can take it to be $t_{\mu}=(1,0,0,0)$, and the extended helicity operator becomes the usual helicity. We use the usual helicity operator to obtain four distinct solutions.

On the other hand, for the transverse equation, taking $p_{\mu}$ in the direction of $t_{\mu}$ has different consequences. Observing Eq. (52),

$$
\left(\sigma^{\mu \nu} P_{\mu} P_{\nu}\right) \psi_{\perp}=s_{-}(\gamma \cdot P)\left(\mathbf{1}-\gamma^{5}\right) i \partial_{\tau} \psi_{\perp}+s_{+} \frac{M}{2}(\gamma \cdot P)\left(\mathbf{1}+\gamma^{5}\right) \psi_{\perp}
$$


then $\sigma^{\mu \nu} P_{\mu} P_{\nu}=0$, and $p_{\perp}^{2}=0$. Considering the free solutions, there is no $\tau$ evolution,

$$
0=s_{+} \frac{M}{2}(\gamma \cdot P)\left(\mathbf{1}+\gamma^{5}\right) \psi_{\perp}
$$

and we can say that $\psi_{\perp R}$ and $\varphi_{\perp L}$ are zero, there is no auxiliary field, and no transverse first order equations exist. All the evolution is described by the longitudinal equation.

It seems that as $p_{\mu}$ departs from the direction of $t_{\mu}$, we depart from the Dirac description, and the event is described by two independent equations of motion, transverse and longitudinal. The event evolution in $\tau$ becomes different for the two versions, differing in phase and spinor content. At the same time the auxiliary fields are spontaneously generated in the transverse case, and from the Dirac field in the longitudinal case. Furthermore, the auxiliary field, treated as a mathematical convenience, but seen to be strongly related to the main field, Eq. (177), may be a further indication of the amount of departure from the on shell Dirac theory.

\section{Conclusions}

Accepting a second order spin equation of the form of the Schrödinger-Stueckelberg equation as the basic structure of the theory, and requiring that the free solutions of a first order equation be also the solutions of the second order one, lead to transverse and longitudinal equations. After a selection process we chose the most suitable forms for the ingredients of these equations. We introduced the induced representations on a time-like vector $t_{\mu}$ and an auxiliary field so that the theory be consistent. The evolution of the free event is governed by two complementary equations, the transverse and longitudinal. We manage to unify the complementary behavior of the event, transverse and longitudinal, with the use of a product Hilbert space. Then, after introducing the electromagnetic coupling, we obtain the correct gyromagnetic ratio, and the second order charged equation describing the evolution of the main field, is fully Hermitian under the scalar product. Finally, we showed how the theory goes over to the Dirac theory in the limit in which $p_{\mu}$ is in the direction of $t_{\mu}$ on mass shell. In this limit the theory changes its structure. The first order transverse equation no longer exists; its auxiliary field and $\tau$ evolution disappear, and the longitudinal equation takes on a new meaning, leading to the original Dirac equation. We also display, in Appendix B, the solutions of the equations, which are characterized by the "extended helicity" and "chiral precedence" 
for the transverse case, and by "extended helicity" and "extended parity" in the longitudinal case. Both cases have the same properties under the generalized parity, charge conjugation, and $\tau$ reversal transformations (as shown in Appendix (D). The objects which transform one into the other under these transformations are pure left or right handed spinors, thus exhibiting the chiral nature of the theory. 


\section{A Idempotents and Nilpotents}

\section{A.1 Idempotents}

Let us consider idempotents of the form (we carry out the analysis in a given Lorentz frame, and discuss later the corresponding invariant forms)

$$
P_{i \pm}=\frac{1}{2}\left(\mathbf{1} \pm \Gamma_{i}\right)
$$

where $\Gamma_{i}$ are elements of the Dirac Clifford algebra (see Appendix D). We state some known facts about these matrices [38

$$
\begin{aligned}
\Gamma_{i}^{2} & =\mathbf{1} \\
\Gamma_{i}^{-1} & =\Gamma_{i} \\
\Gamma_{i}^{\dagger} & =\Gamma_{i} \quad(i=1, \ldots, 16)
\end{aligned}
$$

where "†" implies complex conjugate transpose, and for $j \neq k$,

$$
\begin{aligned}
& \Gamma_{j} \Gamma_{k}=\epsilon_{j k} \Gamma_{l} \quad \epsilon_{j k} \in\{1,-1, i,-i\} \\
& \Gamma_{k} \Gamma_{j}=\left(\epsilon_{j k}\right)^{-1} \Gamma_{l}
\end{aligned}
$$

There are fifteen pairs of $P_{i \pm}$. In fact they are equivalent under automorphism. We show this by defining a unitary transformation $T_{j}$ such that

$$
T_{j}=\frac{1}{\sqrt{2}}\left(\mathbf{1}+i \Gamma_{j}\right) ; T_{j}^{-1}=\frac{1}{\sqrt{2}}\left(\mathbf{1}-i \Gamma_{j}\right)=T_{j}^{\dagger}
$$

Using $T_{j}$ we can transform from one $\Gamma$ to another. If $\Gamma_{i}$ and $\Gamma_{j}$ commute we have

$$
\left[\Gamma_{j}, \Gamma_{i}\right]=0 \Rightarrow\left[T_{j}, \Gamma_{i}\right]=0 \Rightarrow T_{j} \Gamma_{i} T_{j}^{-1}=\Gamma_{i}
$$

and because of Eqs. (189), (190) we have (for $i \neq j$ )

$$
\begin{aligned}
{\left[\Gamma_{j}, \Gamma_{i}\right]=0 } & \Rightarrow \Gamma_{i} \Gamma_{j}-\Gamma_{j} \Gamma_{i}=\left(\epsilon_{i j}-\epsilon_{i j}^{-1}\right) \Gamma_{k}=0 \\
& \Rightarrow \epsilon_{i j}=\epsilon_{i j}^{-1} \Rightarrow \epsilon_{i j}= \pm 1
\end{aligned}
$$

and

$$
\left[\Gamma_{i}, \Gamma_{j}\right]=0 \Rightarrow\left\{\Gamma_{i}, \Gamma_{j}\right\} \neq 0
$$


$\Gamma_{i}$ is invariant under the transformation $T_{j}$; we find a triplet $\Gamma_{i}, \Gamma_{j}, \Gamma_{k}$ which commute among themselves, where $\Gamma_{k}=\Gamma_{i} \Gamma_{j}$. These triplets form maximal commuting sets.

On the other hand, if $\Gamma_{i}$ and $\Gamma_{j}$ do not commute then

$$
\begin{aligned}
{\left[\Gamma_{j}, \Gamma_{i}\right] \neq 0 } & \Rightarrow\left[T_{j}, \Gamma_{i}\right] \neq 0 \Rightarrow\left(\epsilon_{i j}-\epsilon_{i j}^{-1}\right) \Gamma_{k} \neq 0 \\
& \Rightarrow \epsilon_{i j} \neq \epsilon_{i j}^{-1} \Rightarrow \epsilon_{i j}= \pm i
\end{aligned}
$$

This means that for the non-commuting case we have

$$
\left[\Gamma_{j}, \Gamma_{i}\right]= \pm 2 i \Gamma_{k}
$$

and so

$$
T_{j} \Gamma_{i} T_{j}^{-1}=\frac{1}{2}\left(\Gamma_{i}+i\left[\Gamma_{j}, \Gamma_{i}\right]+\Gamma_{j} \Gamma_{i} \Gamma_{j}\right)=\mp \Gamma_{k}
$$

Furthermore we find that

$$
\left[\Gamma_{i}, \Gamma_{j}\right] \neq 0 \Rightarrow\left\{\Gamma_{i}, \Gamma_{j}\right\}=0
$$

We can transform $\Gamma_{i}$ into $\Gamma_{k}$ using $\Gamma_{j}$. This is a Pauli algebra structure for the triplet $\Gamma_{i}, \Gamma_{j}, \Gamma_{k}$.

$$
\begin{array}{lll}
{\left[\Gamma_{j}, \Gamma_{i}\right]= \pm 2 i \Gamma_{k}} & ; & \left\{\Gamma_{j}, \Gamma_{i}\right\}=0 \\
{\left[\Gamma_{i}, \Gamma_{k}\right]= \pm 2 i \Gamma_{j}} & ; & \left\{\Gamma_{i}, \Gamma_{k}\right\}=0 \\
{\left[\Gamma_{k}, \Gamma_{j}\right]= \pm 2 i \Gamma_{i}} & ; & \left\{\Gamma_{k}, \Gamma_{j}\right\}=0
\end{array}
$$

From $P_{i \pm}$ and $P_{j \pm}$ from a maximal commuting set, we can form four primitive idempotents

$$
P_{i \pm} P_{j \pm}=\left(\frac{1}{2}\right)^{2}\left(\mathbf{1} \pm \Gamma_{i}\right)\left(\mathbf{1} \pm \Gamma_{j}\right)
$$

We shall denote these by

$$
\tilde{P}_{\alpha}=P_{i \pm} P_{j \pm} \quad(\alpha=1,2,3,4)
$$

where

$$
\begin{aligned}
& \alpha=1 \longrightarrow i+, j+ \\
& \alpha=2 \longrightarrow i+, j- \\
& \alpha=3 \longrightarrow i-, j+ \\
& \alpha=4 \longrightarrow i-, j-
\end{aligned}
$$


The triplets of nontrivial commuting $\Gamma$ 's are (the numbers refer to the index of the matrices, see Appendix D):

$$
\begin{array}{rrrrr}
6,9,16 & 6,4,15 & 6,5,14 & 9,2,13 & 9,3,12 \\
16,8,11 & 16,7,10 & 15,2,11 & 15,3,7 & 10,4,12 \\
2,10,14 & 11,5,12 & 14,3,8 & 13,5,7 & 8,4,13
\end{array}
$$

For building $\tilde{P}_{\alpha}$ 's we can take any pair from a given triplet, and achieve the same result (get the same $\tilde{P}_{\alpha}$ 's for the triplet); this is because

$$
\tilde{P}_{\alpha}=\left(\frac{1}{2}\right)^{2}\left(\mathbf{1} \pm \Gamma_{i} \pm \Gamma_{j} \pm \Gamma_{i} \Gamma_{j}\right)
$$

and

$$
\Gamma_{i} \Gamma_{j}= \pm \Gamma_{k}
$$

Furthermore, each $\Gamma$ appears only in three of the above triplets, and we can transform from one triplet to another in this "trio" of triplets, by a unitary transformation, thus transforming between different sets of $\tilde{P}_{\alpha}$ 's. If we denote the trio of triplets in which a $\Gamma$ matrix (say, "a") appears as

$$
\begin{aligned}
& \mathrm{a}, b, c \\
& \text { a }, d, e \\
& \text { a , } f, g
\end{aligned}
$$

then we find that we always have a Pauli type relation of anticommutators among the remaining pairs, for example such as:

$$
\begin{aligned}
& \{b, d\}=\{b, f\}=\{d, f\}=0 \\
& \{b, e\}=\{b, g\}=\{e, g\}=0
\end{aligned}
$$

here we took "b" as the "pivot" (i.e. "b" is used for $T_{b}$ ). This enables us to take $T_{b}$ and transform the second triplet into the third: $a, d, e \rightarrow a, f, g$ (it can be done equally well with "c" as a "pivot"), thus getting a transformed set of $\tilde{P}_{\alpha}$ 's. This way we can take a triplet in a trio, and use it to transform between the other two triplets. Looking at the existing triplets we see that we can transform from any triplet to any other by a suitable choice of unitary transformations, i.e., all sets of $\tilde{P}_{\alpha}$ 's are equivalent up to a unitary transformation. 


\section{A.2 Nilpotents}

We can produce non-primitive level nilpotents by taking

$$
\begin{aligned}
& n_{i j}=\left(\Gamma_{i} \pm i \Gamma_{j}\right) \\
& n_{i j}^{2}= \pm i\left\{\Gamma_{i}, \Gamma_{j}\right\}
\end{aligned}
$$

and requiring that $\left\{\Gamma_{i}, \Gamma_{j}\right\}=0$.

Now we turn to primitive level nilpotents. In a certain representation we have $\tilde{P}_{k}=$ $e_{k k},(k=1,2,3,4)$ where $e_{k k}$ is the matrix which has a " 1 " in the $k^{\text {th }}$ row and $k^{\text {th }}$ column, and all other places are zero. Since the matrices $e_{n m},(n \neq m)$ are nilpotent, (we have twelve of these), we get for any Dirac matrix $A$ :

$$
e_{i i} A e_{k k}=\alpha_{i k} e_{i k}
$$

where $\alpha_{i k}$ is the element on the $i^{t h}$ row, $k^{t h}$ column, in $A$. Once we have these twelve nilpotents, others can be built from them. If we want to represent the nilpotents with the help of the $\Gamma$ matrices we need to have

$$
\begin{aligned}
& N_{i k j}=\tilde{P}_{i} \Gamma_{k} \tilde{P}_{j} \\
& N_{i k j}^{2}=\tilde{P}_{i} \Gamma_{k} \tilde{P}_{j} \tilde{P}_{i} \Gamma_{k} \tilde{P}_{j}=0 \quad(i \neq j)
\end{aligned}
$$

this is because $\tilde{P}_{j} \tilde{P}_{i}=0$ always. We must guard against $N_{i k j}$ being zero, therefore we must not have $\left[\tilde{P}_{i}, \Gamma_{k}\right]=0$ or $\left[\tilde{P}_{j}, \Gamma_{k}\right]=0$ and $i \neq j$. In this case, for $i=j$, the representation is diagonal. Actually if we take a closer look at $\tilde{P}_{i}, \tilde{P}_{j}$, for example,

$$
\tilde{P}_{i}=P_{n \pm} P_{m \pm} \quad \tilde{P}_{j}=P_{n \pm} P_{m \mp}
$$

then if $\left\{\Gamma_{k}, \Gamma_{m}\right\}=0, \Gamma_{k} P_{m \pm}=P_{m \mp} \Gamma_{k}$, and $P_{m}$ flips sign. We can summarize the nonzero useful nilpotents as follows:

1.

$$
\begin{gathered}
\left\{\Gamma_{k}, \Gamma_{m}\right\}=0 \quad ; \quad\left[\Gamma_{k}, \Gamma_{n}\right]=0 \Longrightarrow \\
\tilde{P}_{i} \Gamma_{k} \tilde{P}_{j}=P_{n \pm} P_{m \pm} \Gamma_{k} P_{n \pm} P_{m \mp}=\Gamma_{k} P_{n \pm} P_{m \mp}=\Gamma_{k} \tilde{P}_{j} \\
\tilde{P}_{i^{\prime}} \Gamma_{k} \tilde{P}_{j^{\prime}}=P_{n \mp} P_{m \pm} \Gamma_{k} P_{n \mp} P_{m \mp}=\Gamma_{k} P_{n \mp} P_{m \mp}=\Gamma_{k} \tilde{P}_{j^{\prime}}
\end{gathered}
$$




$$
\begin{gathered}
\left\{\Gamma_{k}, \Gamma_{m}\right\}=0 \quad ;\left\{\Gamma_{k}, \Gamma_{n}\right\}=0 \Longrightarrow \\
\tilde{P}_{i} \Gamma_{k} \tilde{P}_{j}=P_{n \pm} P_{m \pm} \Gamma_{k} P_{n \mp} P_{m \mp}=\Gamma_{k} P_{n \mp} P_{m \mp}=\Gamma_{k} \tilde{P}_{j} \\
\tilde{P}_{i^{\prime}} \Gamma_{k} \tilde{P}_{j^{\prime}}=P_{n \pm} P_{m \mp} \Gamma_{k} P_{n \mp} P_{m \pm}=\Gamma_{k} P_{n \mp} P_{m \pm}=\Gamma_{k} \tilde{P}_{j^{\prime}}
\end{gathered}
$$

thus showing that there are $N_{i k j} \neq 0$. The first entry results in four nilpotents which are equivalent to the four generated by the second entry up to a sign. Since both cases are equivalent we shall concentrate on the second case. We find that any two commuting $\Gamma$ 's, have exactly four mutual anticommuting $\Gamma$ 's in common. Taking the two commuting $\Gamma$ 's as a pair from a triplet, then for each pair the four anticommuting $\Gamma$ 's are distinct. Therefore we get a "structure" which can be illustrated by a specific example; for the triplet $P_{2}, P_{11}, P_{15}$ the relevant structure is :

$$
\begin{array}{lll}
P_{11}, P_{15} & : & \Gamma_{9}, \Gamma_{10}, \Gamma_{13}, \Gamma_{14} \\
P_{2}, P_{11} & : & \Gamma_{3}, \Gamma_{4}, \Gamma_{6}, \Gamma_{7} \\
P_{15}, P_{2} & : & \Gamma_{5}, \Gamma_{8}, \Gamma_{12}, \Gamma_{16}
\end{array}
$$

The $P$ 's can be thought of as idempotent generators, and the $\Gamma$ 's as nilpotent generators. The structure covers all $\Gamma$ 's. From the two $P$ 's in a row we can build the four $\tilde{P}_{\alpha}$ 's. We may take any $\Gamma$ from that specific row to produce the four nilpotents (all $\Gamma$ 's in a row produce the same nilpotents). The three rows produce four distinct nilpotents each, summing up to the basic twelve required for $4 \times 4$ matrices. The important thing to remember is that by a unitary transformation we can transform from one triplet to another, and thus from one structure to another, hence all structures are equivalent up to a unitary transformation. A special feature of the above structure $\left(P_{2}, P_{11}, P_{15}\right)$ is that it produces the idempotents and nilpotents characterized by the matrices $e_{i j}$.

\section{A.3 Nilpotents for $N_{ \pm}$}

Since we are looking for $N_{-}$which gives us a positive definite probability density, Eq. (37), we need $N_{-}$to include an "anchor" in the form of $\gamma^{0}$, which assures a positive part, and up to one more term (which should be smaller or equal to the anchor since $\gamma$ matrices are orthogonal). Now, considering the form of the currents in Eq. (37), $\gamma^{0}=\Gamma_{2}$ 
is the only anchor possible because of the appearance of $\gamma^{0}$ in $\bar{\psi}$, so we have to look at the row in the structure where $\Gamma_{2}$ resides and calculate the nilpotents (all other rows will not produce nilpotents with this anchor). We take for example the structure:

$$
\begin{array}{lll}
P_{6}, P_{16} & : & \Gamma_{2}, \Gamma_{3}, \Gamma_{12}, \Gamma_{13} \\
P_{6}, P_{9} & : & \Gamma_{7}, \Gamma_{8}, \Gamma_{10}, \Gamma_{11} \\
P_{9}, P_{16} & : & \Gamma_{4}, \Gamma_{5}, \Gamma_{14}, \Gamma_{15}
\end{array}
$$

and focus on the first row. Now we generate nilpotents $N_{i},(i=1,2,3,4)$

$$
\begin{aligned}
& N_{1}=\Gamma_{2} P_{6-} P_{16+}=\left(\frac{1}{2}\right)^{2}\left(\Gamma_{2}+i \Gamma_{3}+i \Gamma_{12}-\Gamma_{13}\right) \\
& N_{2}=\Gamma_{2} P_{6-} P_{16-}=\left(\frac{1}{2}\right)^{2}\left(\Gamma_{2}+i \Gamma_{3}-i \Gamma_{12}+\Gamma_{13}\right) \\
& N_{3}=\Gamma_{2} P_{6+} P_{16+}=\left(\frac{1}{2}\right)^{2}\left(\Gamma_{2}-i \Gamma_{3}+i \Gamma_{12}+\Gamma_{13}\right) \\
& N_{4}=\Gamma_{2} P_{6+} P_{16-}=\left(\frac{1}{2}\right)^{2}\left(\Gamma_{2}-i \Gamma_{3}-i \Gamma_{12}-\Gamma_{13}\right)
\end{aligned}
$$

In terms of the $\gamma$ matrices we get

$$
\begin{aligned}
& N_{1}=\left(\frac{1}{2}\right)^{2}\left(\gamma^{0}-\gamma^{1}+\gamma^{0} \gamma^{5}+\gamma^{1} \gamma^{5}\right) \\
& N_{2}=\left(\frac{1}{2}\right)^{2}\left(\gamma^{0}-\gamma^{1}-\gamma^{0} \gamma^{5}-\gamma^{1} \gamma^{5}\right) \\
& N_{3}=\left(\frac{1}{2}\right)^{2}\left(\gamma^{0}+\gamma^{1}+\gamma^{0} \gamma^{5}-\gamma^{1} \gamma^{5}\right) \\
& N_{4}=\left(\frac{1}{2}\right)^{2}\left(\gamma^{0}+\gamma^{1}-\gamma^{0} \gamma^{5}+\gamma^{1} \gamma^{5}\right)
\end{aligned}
$$

We consider these results as given in a specific reference frame. The Lorentz invariant form of these nilpotents is:

$$
\begin{aligned}
& N_{1}^{\prime}=\left(\gamma \cdot l^{(1-)}\right)+\left(\gamma \cdot l^{(1+)}\right) \gamma^{5} \\
& N_{2}^{\prime}=\left(\gamma \cdot l^{(1-)}\right)-\left(\gamma \cdot l^{(1+)}\right) \gamma^{5} \\
& N_{3}^{\prime}=\left(\gamma \cdot l^{(1+)}\right)+\left(\gamma \cdot l^{(1-)}\right) \gamma^{5} \\
& N_{4}^{\prime}=\left(\gamma \cdot l^{(1+)}\right)-\left(\gamma \cdot l^{(1-)}\right) \gamma^{5}
\end{aligned}
$$

where we used for the four-vectors $l^{(1 \pm)}$ the notation

$$
\begin{aligned}
& l^{(1-)}=\left(l_{0},-l_{0}, 0,0\right) \\
& l^{(1+)}=\left(l_{0}, l_{0}, 0,0\right)
\end{aligned}
$$


and $l_{0}$ is some number.

The requirement for a positive definite probability density implies a reduction in half of the participating matrices in each nilpotent combination of Eqs. (211); we can do this by reverting to the non-primitive level nilpotents:

$$
\begin{array}{ll}
N_{1}+N_{2}=\frac{1}{2}\left(\gamma^{0}-\gamma^{1}\right) & \rightarrow\left(\gamma \cdot l^{(1-)}\right) \\
N_{1}-N_{2}=\frac{1}{2}\left(\gamma^{0} \gamma^{5}+\gamma^{1} \gamma^{5}\right) & \rightarrow\left(\gamma \cdot l^{(1+)}\right) \gamma^{5} \\
N_{1}+N_{3}=\frac{1}{2}\left(\gamma^{0}+\gamma^{0} \gamma^{5}\right) & \rightarrow(\gamma \cdot t)\left(\mathbf{1}+\gamma^{5}\right) \\
N_{1}-N_{3}=\frac{1}{2}\left(-\gamma^{1}+\gamma^{1} \gamma^{5}\right) & \rightarrow-\left(\gamma \cdot s^{(1)}\right)\left(\mathbf{1}-\gamma^{5}\right) \\
N_{3}+N_{4}=\frac{1}{2}\left(\gamma^{0}+\gamma^{1}\right) & \rightarrow\left(\gamma \cdot l^{(1+)}\right) \\
N_{3}-N_{4}=\frac{1}{2}\left(\gamma^{0} \gamma^{5}-\gamma^{1} \gamma^{5}\right) & \rightarrow\left(\gamma \cdot l^{(1-)}\right) \gamma^{5} \\
N_{2}+N_{4}=\frac{1}{2}\left(\gamma^{0}-\gamma^{0} \gamma^{5}\right) & \rightarrow(\gamma \cdot t)\left(\mathbf{1}-\gamma^{5}\right) \\
N_{2}-N_{4}=\frac{1}{2}\left(-\gamma^{1}-\gamma^{1} \gamma^{5}\right) & \rightarrow-\left(\gamma \cdot s^{(1)}\right)\left(\mathbf{1}+\gamma^{5}\right)
\end{array}
$$

where the four-vectors $s^{(1)}$, and $t$ have been used

$$
\begin{aligned}
t & =\left(t_{0}, 0,0,0\right) \\
s^{(1)} & =\left(0, s_{1}, 0,0\right)
\end{aligned}
$$

and $t_{0}, s_{1}$ are numbers. Going to non-primitive level nilpotents, we get twice as many nilpotents (eight), which come in pairs differing in sign. Each pair can be a good candidate for $N_{+}, N_{-}$. From the nilpotents in Eqs. (214), only $\left(\gamma \cdot l^{(1 \pm)}\right)$ and $(\gamma \cdot t)\left(\mathbf{1} \pm \gamma^{5}\right.$ ) are valid for a positive definite probability density (having $(\gamma \cdot t)$ as a Lorentz covariant positive definite "anchor"). Furthermore, it seems that $\left(\gamma \cdot l^{(1 \pm)}\right)$ and $(\gamma \cdot t)\left(\mathbf{1} \pm \gamma^{5}\right)$ should not be treated equivalently, since they are derived from the same row in the same structure.

Carrying out the procedure of finding nilpotents on the twelve available structures, (there are fifteen triplets, but three of them include $\Gamma_{2}$ as an idempotent generator instead of a nilpotent generator), we find the following nilpotents. We mention only the relevant row in the structure containing $\Gamma_{2}$, and giving rise to nilpotents which have a 
chance of being positive definite. The generating idempotents are in brackets

$$
\begin{aligned}
& P_{9},\left(P_{6}, P_{16}\right) \quad: \quad \Gamma_{2}, \Gamma_{3}, \Gamma_{12}, \Gamma_{13} \rightarrow\left(\gamma \cdot l^{(1 \pm)}\right), \quad(\gamma \cdot t)\left(\mathbf{1} \pm \gamma^{5}\right) \\
& P_{15},\left(P_{4}, P_{6}\right): \Gamma_{2}, \Gamma_{3}, \Gamma_{7}, \Gamma_{11} \quad \rightarrow \quad\left(\gamma \cdot l^{(1 \pm)}\right), \quad(\gamma \cdot t) \pm \sigma t s^{(2)} \\
& P_{14},\left(P_{5}, P_{6}\right) \quad: \quad \Gamma_{2}, \Gamma_{3}, \Gamma_{8}, \Gamma_{16} \quad \rightarrow \quad\left(\gamma \cdot l^{(1 \pm)}\right), \quad(\gamma \cdot t) \pm \sigma t s^{(3)} \\
& P_{9},\left(P_{3}, P_{12}\right) \quad: \quad \Gamma_{2}, \Gamma_{6}, \Gamma_{13}, \Gamma_{16} \quad \rightarrow \quad(\gamma \cdot t) \pm \sigma t s^{(1)}, \quad(\gamma \cdot t) \pm i \gamma^{5} \\
& P_{11},\left(P_{8}, P_{16}\right): \Gamma_{2}, \Gamma_{5}, \Gamma_{12}, \Gamma_{15} \rightarrow\left(\gamma \cdot l^{(3 \pm)}\right), \quad(\gamma \cdot t)\left(\mathbf{1} \pm \gamma^{5}\right) \\
& P_{10},\left(P_{7}, P_{16}\right): \Gamma_{2}, \Gamma_{4}, \Gamma_{12}, \Gamma_{14} \rightarrow\left(\gamma \cdot l^{(2 \pm)}\right), \quad(\gamma \cdot t)\left(\mathbf{1} \pm \gamma^{5}\right) \\
& P_{15},\left(P_{3}, P_{7}\right) \quad: \quad \Gamma_{2}, \Gamma_{4}, \Gamma_{6}, \Gamma_{11} \quad \rightarrow \quad(\gamma \cdot t) \pm \sigma t s^{(1)}, \quad\left(\gamma \cdot l^{(2 \pm)}\right) \\
& P_{10},\left(P_{4}, P_{12}\right): \Gamma_{2}, \Gamma_{7}, \Gamma_{14}, \Gamma_{16} \rightarrow(\gamma \cdot t) \pm \sigma t s^{(2)}, \quad(\gamma \cdot t) \pm i \gamma^{5} \\
& P_{11},\left(P_{5}, P_{12}\right): \Gamma_{2}, \Gamma_{8}, \Gamma_{15}, \Gamma_{16} \rightarrow(\gamma \cdot t) \pm \sigma t s^{(3)}, \quad(\gamma \cdot t) \pm i \gamma^{5} \\
& P_{14},\left(P_{3}, P_{8}\right): \Gamma_{2}, \Gamma_{5}, \Gamma_{6}, \Gamma_{10} \quad \rightarrow \quad\left(\gamma \cdot l^{(3 \pm)}\right), \quad(\gamma \cdot t) \pm \sigma t s^{(1)} \\
& P_{13},\left(P_{5}, P_{7}\right) \quad: \quad \Gamma_{2}, \Gamma_{4}, \Gamma_{8}, \Gamma_{9} \quad \rightarrow \quad\left(\gamma \cdot l^{(2 \pm)}\right), \quad(\gamma \cdot t) \pm \sigma t s^{(3)} \\
& P_{13},\left(P_{4}, P_{8}\right) \quad: \quad \Gamma_{2}, \Gamma_{5}, \Gamma_{7}, \Gamma_{9} \quad \rightarrow \quad\left(\gamma \cdot l^{(3 \pm)}\right), \quad(\gamma \cdot t) \pm \sigma t s^{(2)}
\end{aligned}
$$

where

$$
\begin{aligned}
& s^{(2 \pm)}=\left(0,0, \pm s_{2}, 0\right) \\
& s^{(3 \pm)}=\left(0,0,0, \pm s_{3}\right) \\
& l^{(2 \pm)}=\left(l_{0}, 0, \pm l_{0}, 0\right) \\
& l^{(3 \pm)}=\left(l_{0}, 0,0, \pm l_{0}\right)
\end{aligned}
$$

\section{B Appendix - Solutions of the equations}




\section{B.1 Solutions of the transverse equation}

We consider plane wave solutions that satisfy the transverse Schrödinger-Stueckelberg equation as well, of the form

$$
\phi_{\perp \tau t}(p) \sim e^{-i \frac{p_{\perp}^{2}}{2 M} \tau+i p x} u_{\perp}(p, t)
$$

where $u_{\perp}(p, t)$ is a four-spinor dependent on the specific momentum and vector $t_{\mu}$. We observe that Eqs. (64), 65) commute with the operator

$$
h(p, t) \equiv \frac{i}{\left|p_{\perp}\right|}(\sigma p t) \gamma^{5}
$$

This operator was shown by Horwitz and Arshansky [23] to correspond to helicity in the frame where $t_{\mu}=(1,0,0,0)$,

$$
\frac{i}{\left|p_{\perp}\right|}(\sigma p t) \gamma^{5} \longrightarrow \frac{\boldsymbol{\Sigma} \cdot \mathbf{p}}{|\mathbf{p}|}
$$

so we shall call $h(p, t)$ the extended helicity operator. An interesting feature of the extended helicity operator is that in the reference frame where $p_{\mu}=\left(p_{0}, 0,0,0\right)$, we get

$$
\frac{i}{\left|p_{\perp}\right|}(\sigma p t) \gamma^{5} \longrightarrow \frac{\boldsymbol{\Sigma} \cdot \mathbf{t}}{|\mathbf{t}|}
$$

This is because in this frame $\left|p_{\perp}\right|=+p_{0} \sqrt{\mathbf{t}^{2}}$. The operator $h(p, t)$ can be decomposed into two partial operators $h(p, t)=h_{5}(t) \cdot h_{p}(p, t)$ which are

$$
\begin{aligned}
h_{5}(t) & \equiv i(\gamma \cdot t) \gamma^{5} \\
h_{p}(p, t) & \equiv \frac{1}{\left|p_{\perp}\right|}(\sigma p t)(\gamma \cdot t)
\end{aligned}
$$

and where they all commute. The operator $h_{5}(t)$ when looked upon in the frame where $t_{\mu}=(1,0,0,0)$ is seen to be

$$
h_{5}(t) \longrightarrow i \gamma^{0} \gamma^{5}=\left(\begin{array}{cc}
0 & i \\
-i & 0
\end{array}\right)
$$

It exchanges the left and right handed parts of the wave function, and gives them a relative phase factor. The left part is rotated counterclockwise by $\pi / 2$ in the complex plane and the right part is rotated clockwise by the same amount. We call this operator the 
chiral precedence operator because its eigenvalues indicate which chiral part precedes the other relative to a counterclockwise rotation in the complex plane. This is an interesting feature of the solutions of the equations of motion since it expresses an inherent broken symmetry between the left and the right parts of the wave function. Moreover, it gives physical meaning to the relative phase of the wave function's components, and creates a coherence which is preserved through the evolution. This feature of the wave function is independent of its momentum.

We assumed a four-spinor as the basic structure of the solutions, therefore we can use two of these operators to characterize completely the four available solutions. The chiral precedence operator (for this purpose the product of helicity operator and chiral precedence operator has the same effect) also transforms Eq. (52), the equation of motion, into Eq. (60), the equation of motion with interchanged nilpotents, and vice versa, therefore relating $\psi_{\perp}$ to $\varphi_{\perp}$

$$
i(\gamma \cdot t) \gamma^{5} \psi_{\perp}=\varphi_{\perp}
$$

This way, the structure of the main field (and auxiliary field) is set to conform to the eigenvectors of the chiral precedence projection operator

$$
P_{h_{5} \pm}=\frac{1}{2}\left(\mathbf{1} \pm i(\gamma \cdot t) \gamma^{5}\right)
$$

where $P_{h_{5}}^{2}=P_{h_{5} \pm}$. This projection operator is connected to the projection part of one of the nilpotent options discussed in Appendix A Eqs. (40), and is non-equivalent to the nilpotents we use, since we cannot transform from one to the other by a unitary transformation.

The solutions are seen to be

$$
\zeta_{\perp}^{+}(t) \equiv\left(\begin{array}{c}
i \sigma^{\mu} t_{\mu} \xi^{+} \\
\xi^{+}
\end{array}\right) \quad \zeta_{\perp}^{-}(t) \equiv\left(\begin{array}{c}
\xi^{-} \\
i \tilde{\sigma}^{\mu} t_{\mu} \xi^{-}
\end{array}\right)
$$

where the superscript \pm denotes the eigenvalue of the eigenvectors when operated upon by the chiral precedence operator. When $P_{h_{5}+}$ operates on $\zeta_{\perp}^{+}(t)$ it gives 1 , and on $\zeta_{\perp}^{-}(t)$ it gives 0 . The opposite happens for $P_{h_{5}-}$. In the special frame where $t_{\mu}=(1,0,0,0)$ the solutions are

$$
\zeta_{\perp}^{+}=\left(\begin{array}{c}
i \xi^{+} \\
\xi^{+}
\end{array}\right) \quad \zeta_{\perp}^{-}=\left(\begin{array}{c}
\xi^{-} \\
i \xi^{-}
\end{array}\right)
$$


and $\xi^{ \pm}$can be taken as two independent vectors, for example

$$
\xi^{+}=\left(\begin{array}{l}
1 \\
0
\end{array}\right) \quad \xi^{-}=\left(\begin{array}{l}
0 \\
1
\end{array}\right)
$$

Since each spinor can be decomposed and characterized by the helicity projection operator

$$
P_{h \pm}=\frac{1}{2}\left(\mathbf{1} \pm \frac{i}{\left|p_{\perp}\right|}(\sigma p t) \gamma^{5}\right)
$$

where $P_{h \pm}^{2}=P_{h \pm}$, and by the chiral precedence operator, we use them to characterize the four available solutions for a specific momentum.

In order to find the general form of the solutions, we consider the projection operators $P_{h \pm}$ and $P_{h_{5} \pm}$ which commute. We can form the following non-normalized solutions

$$
u_{\perp}^{r s}(p, t)=\frac{1}{2}\left(\mathbf{1}+\epsilon_{s} \frac{i}{\left|p_{\perp}\right|}(\sigma p t) \gamma^{5}\right) \zeta_{\perp}^{r}(t)
$$

where $\zeta_{\perp}^{r}(t)$ are boosted four-spinors composed of stacked $\xi^{ \pm}$two-spinors as in Eq. (228), and $\epsilon_{s}$ is \pm 1 depending on the eigenvalues of the extended helicity operator. We define the normalization constant $N(p, t)$ so that

$$
N^{2}(p, t) \bar{u}_{\perp}^{r^{\prime} s^{\prime}}(p, t)(\gamma \cdot t) u_{\perp}^{r s}(p, t)=\delta_{r r^{\prime}} \delta_{s s^{\prime}}
$$

We turn now to compute the total integrated probability density, which should be unity.

$$
\int d^{4} x \rho_{\perp \tau t}(x)=\int d^{4} x\left(\bar{\phi}_{\perp \tau t}(x)(\gamma \cdot t) \phi_{\perp \tau t}(x)\right)
$$

A wave packet is

$$
\phi_{\perp \tau t}(x)=\int d^{4} p N(p, t) \sum_{r s} c\left(r, s, p^{2}, p \cdot t\right) u_{\perp}^{r s}(p, t) e^{-i \frac{p_{\perp}^{2}}{2 M} \tau+i p x}
$$

where $c\left(r, s, p^{2}, p \cdot t\right)$ are the weights of the wave packet's components. Performing the $d^{4} x$ integration in Eq. (233) and one $d^{4} p$ integration, we obtain

$$
\begin{aligned}
\int d^{4} x \rho_{\perp \tau t}(x)= & (2 \pi)^{4} \int d^{4} p N^{2}(p, t) \sum_{r, r^{\prime}, s, s^{\prime}} c^{*}\left(r^{\prime}, s^{\prime}, p^{2}, p \cdot t\right) c\left(r, s, p^{2}, p \cdot t\right) \\
& \times \bar{u}_{\perp}^{r^{\prime} s^{\prime}}(p, t)(\gamma \cdot t) u_{\perp}^{r s}(p, t)
\end{aligned}
$$

Using Eq. (232), we find (one can say that Eq. (236) results in a constant depending on $t_{\mu}$ rather than 1 , and the integration over $d^{4} t \delta\left(t^{2}+1\right)$ is normalized to 1$)$

$$
\int d^{4} x \rho_{\perp \tau t}(x)=(2 \pi)^{4} \int d^{4} p \sum_{r, s}\left|c\left(r, s, p^{2}, p \cdot t\right)\right|^{2}=1
$$


We compute the total integrated currents on all space-time and all $t_{\mu}$, from Eq. (93), by using $j_{\perp \tau}^{\mu}=-\frac{i}{2 M}\left(\bar{\phi}_{\perp \tau t}(\gamma \cdot t) \stackrel{\leftrightarrow}{\partial_{\perp}^{\mu}} \phi_{\perp \tau t}\right)$. Then we have

$$
\begin{aligned}
J_{\perp \mu}=\int d^{4} x d^{4} t \delta\left(t^{2}+1\right) j_{\perp \tau \mu}(x) & =\int d^{4} x d^{4} t \delta\left(t^{2}+1\right) \frac{-i}{2 M}\left(\bar{\phi}_{\perp \tau t}(x)(\gamma \cdot t) \stackrel{\leftrightarrow}{\perp \mu}_{\perp \tau t}(x)\right) \\
& =(2 \pi)^{4} \int d^{4} p d^{4} t \delta\left(t^{2}+1\right) \frac{p_{\perp \mu}}{M} \sum_{r, s}\left|c\left(r, s, p^{2}, p \cdot t\right)\right|^{2}
\end{aligned}
$$

so the integrated current is

$$
J_{\perp \mu}=\left\langle\frac{p_{\perp \mu}}{M}\right\rangle
$$

Now, $p_{\perp \mu}$ is space-like, and so is $j_{\perp \mu}$. Since we perform the integration over $t_{\mu}$, thus having various components of $p_{\mu}$, it is possible to acquire a time-like $J_{\perp \mu}$ current, as in 32 .

The equations of motion, Eqs. (64), (65), describe the evolution of the state of a four dimensional momentum and currents, existing in the plane orthogonal to the time-like vector $t_{\mu}$. Considering the frame where $t_{\mu}=(1,0,0,0), p_{\perp \mu}$ becomes $p_{i}$ and we are dealing with a space-like current. The appearance of $P_{\mu}$ as a four-vector in the theory is purely for reasons of keeping the invariance of the theory. Another manifestation of this aspect is presented in Section VII, where we find the Hermitian term $\sigma_{t}^{\mu \nu} f_{\mu \nu}$, which transforms to $\sigma^{i j}$ in the frame where $t_{\mu}=(1,0,0,0)$, and that is why there is no problem of Hermiticity in the second order charged equation. The momentum of an event can be thought of as being partitioned by $t_{\mu}$ into a longitudinal part and a transverse part. Each part evolves according to a different evolution equation, and they refer to the same vectors $p_{\mu}, t_{\mu}$.

\section{B.2 Solutions of the longitudinal equation}

For the longitudinal equation we consider the plane wave solutions of the form

$$
\phi_{\| \tau t}(p) \sim e^{-i \frac{p_{\|}^{2}}{2 M} \tau+i p x} u_{\|}(p, t)
$$

Again the equations of motion, Eqs. (69),(70) commute with the helicity operator from Eq. (219), so it can be used to characterize the solutions. Two more operators that 
commute with the equations of motion are

$$
\begin{aligned}
h_{t}(t) & \equiv(\gamma \cdot t) \\
h_{p t 5}(p, t) & \equiv(\gamma \cdot t) \frac{i}{\left|p_{\perp}\right|}(\sigma p t) \gamma^{5}
\end{aligned}
$$

where $h_{p t 5}=h_{t}(p, t) \cdot h(p, t)$, and they all commute. Notice that

$$
\begin{aligned}
h_{t}(t) & =i \gamma^{5} h_{5}(t) \\
h_{p t 5}(p, t) & =i \gamma^{5} h_{p}(p, t)
\end{aligned}
$$

The characterization is by the projection of helicity Eq. (230) and the projection operator

$$
P_{h_{t} \pm}=\frac{1}{2}(\mathbf{1} \pm(\gamma \cdot t))
$$

The operator $h_{t}(t)$ in the frame where $t_{\mu}=(1,0,0,0)$ is seen to be just $\gamma^{0}$ which is the parity operator; we denote it as the extended parity operator. Therefore the characterization of the solutions of the longitudinal equation is done by extended helicity and extended parity. The extended parity operator also transforms Eq. (66) into its counterpart with nilpotents exchanged, and vice versa. We obtain the relation

$$
(\gamma \cdot t) \psi_{\|}=\varphi_{\|}
$$

The solutions are seen to be

$$
\zeta_{\|}^{+}(t) \equiv\left(\begin{array}{c}
\sigma^{\mu} t_{\mu} \xi^{+} \\
\xi^{+}
\end{array}\right) \quad \zeta_{\|}^{-}(t) \equiv\left(\begin{array}{c}
\xi^{-} \\
-\tilde{\sigma}^{\mu} t_{\mu} \xi^{-}
\end{array}\right)
$$

where the superscript \pm denotes the eigenvalue of the eigenvectors when operated upon by the extended parity operator. In the special frame where $t_{\mu}=(1,0,0,0)$ the solutions are

$$
\zeta_{\|}^{+}=\left(\begin{array}{c}
\xi^{+} \\
\xi^{+}
\end{array}\right) \quad \zeta_{\|}^{-}=\left(\begin{array}{c}
\xi^{-} \\
-\xi^{-}
\end{array}\right)
$$

and $\xi^{ \pm}$can be taken as two independent vectors. The general form of the non-normalized spinor solutions is

$$
u_{\|}^{r s}(p, t)=\frac{1}{2}\left(\mathbf{1}+\epsilon_{s} \frac{i}{\left|p_{\perp}\right|}(\sigma p t) \gamma^{5}\right) \zeta_{\|}^{r}(t)
$$

where $\epsilon_{s}$ has the same meaning as in the transverse case, and $r$ denotes the eigenvalues of extended parity. 
As for the transverse case we set the normalization condition on the probability density

$$
\int d^{4} x \rho_{\| \tau t}(x)=\int d^{4} x\left(\bar{\phi}_{\| \tau t}(x)(\gamma \cdot t) \phi_{\| \tau t}(x)\right)
$$

so that

$$
N^{2}(p, t) \bar{u}_{\|}^{r^{\prime} s^{\prime}}(p, t)(\gamma \cdot t) u_{\|}^{r s}(p, t)=\delta_{r r^{\prime}} \delta_{s s^{\prime}}
$$

A wave packet is

$$
\phi_{\| \tau t}(x)=\int d^{4} p N(p, t) \sum_{r, s} d\left(r, s, p^{2}, p \cdot t\right) u_{\|}^{r s}(p, t) e^{-i \frac{p_{\|}^{2}}{2 M} \tau+i p x}
$$

and we get after the integration over $d^{4} x$ and one over $d^{4} p$

$$
\begin{aligned}
\int d^{4} x \rho_{\| \tau t}(x)= & (2 \pi)^{4} \int d^{4} p N^{2}(p, t) \sum_{r, r^{\prime}, s, s^{\prime}} d^{*}\left(r^{\prime}, s^{\prime}, p^{2}, p \cdot t\right) d\left(r, s, p^{2}, p \cdot t\right) \\
& \times \bar{u}_{\|}^{r^{\prime} s^{\prime}}(p, t)(\gamma \cdot t) u_{\|}^{r s}(p, t)
\end{aligned}
$$

Using Eq. (248) we find

$$
\int d^{4} x \rho_{\| \tau t}(x)=(2 \pi)^{4} \int d^{4} p \sum_{r, s}\left|d\left(r, s, p^{2}, p \cdot t\right)\right|^{2}=1
$$

As for the integrated currents using, $j_{\| \tau}^{\mu}=-\frac{i}{2 M}\left(\bar{\phi}_{\|}(\gamma \cdot t) \overleftrightarrow{\partial_{\|}^{\mu}} \phi_{\|}\right)$, we find

$$
\begin{aligned}
J_{\| \mu}=\int d^{4} x d^{4} t \delta\left(t^{2}+1\right) j_{\| \tau \mu}(x) & =\int d^{4} x d^{4} t \delta\left(t^{2}+1\right) \frac{-i}{2 M}\left(\bar{\phi}_{\| \tau t}(x)(\gamma \cdot t) \stackrel{\leftrightarrow}{\partial_{\| \mu}} \phi_{\| \tau t}(x)\right) \\
& =(2 \pi)^{4} \int d^{4} p d^{4} t \delta\left(t^{2}+1\right) \frac{p_{\| \mu}}{M} \sum_{r, r^{\prime}, s, s^{\prime}}\left|d\left(r, s, p^{2}, p \cdot t\right)\right|^{2}
\end{aligned}
$$

so the integration of the current (over $t_{\mu}$ as well) is

$$
J_{\| \mu}=\left\langle\frac{p_{\| \mu}}{M}\right\rangle
$$

In a frame where $t_{\mu}=(1,0,0,0)$, the zeroth component of the currents $j_{\| \mu}$ is the only one which survives due to the vanishing of $\partial_{\perp i}$ in this frame, so the currents are timelike. In accordance with Lorentz invariance this is true in any reference frame. As in the transverse case, $J_{\| \mu}$ may be time-like, depending on the relative weights of the wave packet's components. 


\section{Appendix - Discrete symmetries}

When dealing with the discrete symmetries of the theory it is necessary to know how the pre-Maxwell field $a_{\alpha},(\alpha=0,1,2,3, \tau)$ transforms. We require that the free kinetic term

of the pre-Maxwell field (see [27]), $\frac{1}{4} \lambda f^{\alpha \beta} f_{\alpha \beta}$, be invariant. Furthermore, we define some new forms of solutions for both the transverse and longitudinal equations, which are just the ones defined in Appendix B but transformed, and are helpful for a full discussion. The basic form of the solutions of the transverse equations are given in Eq. (227); we redefine them by choosing $\xi^{ \pm}$to be an eigenvector of $-i \sigma^{2}$ with eigenvalues of $\pm i$

$$
\xi^{+}=\left(\begin{array}{c}
i \\
1
\end{array}\right) \quad \xi^{-}=\left(\begin{array}{l}
1 \\
i
\end{array}\right)
$$

Multiplying each component of $\zeta_{\perp}^{+}(t)$ by $-i \tilde{\sigma}^{\mu} t_{\mu}$, and each component of $\zeta_{\perp}^{-}(t)$ by $-i \sigma^{\mu} t_{\mu}$, and exchanging $\xi^{+}$by $\xi^{-}$, we get

$$
\eta_{\perp}^{+}(t) \equiv\left(\begin{array}{c}
\xi^{-} \\
-i \tilde{\sigma}^{\mu} t_{\mu} \xi^{-}
\end{array}\right) \eta_{\perp}^{-}(t) \equiv\left(\begin{array}{c}
-i \sigma^{\mu} t_{\mu} \xi^{+} \\
\xi^{+}
\end{array}\right)
$$

The basic form of the solutions of the longitudinal equations is given in Eq. (244), by multiplying both components of $\zeta_{\|}^{+}(t)$ by $\tilde{\sigma}^{\mu} t_{\mu}$, and $\zeta_{\|}^{-}(t)$ by $-\sigma^{\mu} t_{\mu}$, and exchanging $\xi^{+}$ by $\xi^{-}$, we get

$$
\eta_{\|}^{+}(t) \equiv\left(\begin{array}{c}
\xi^{-} \\
\tilde{\sigma}^{\mu} t_{\mu} \xi^{-}
\end{array}\right) \quad \eta_{\|}^{-}(t) \equiv\left(\begin{array}{c}
-\sigma^{\mu} t_{\mu} \xi^{+} \\
\xi^{+}
\end{array}\right)
$$

We now proceed to the treatment of parity, charge conjugation, and $\tau$ reversal.

\section{C.1 Parity}

The transverse charged equations of motion exhibit a symmetry under the inversion of parity. The transformation is

$$
\begin{aligned}
\mathbf{x} & \rightarrow-\mathbf{x} \\
\mathbf{P} & \rightarrow-\mathbf{P} \\
\mathbf{t} & \rightarrow-\mathbf{t} \\
\mathbf{a}(x) & \rightarrow \mathbf{a}\left(x^{\prime}\right)=-\mathbf{a}(x)
\end{aligned}
$$


Applying it to the transverse equations of motion we get

$$
\begin{aligned}
& \left(-\sigma^{0 i} \Pi_{0} t_{i}-\sigma^{i 0} \Pi_{i} t_{0}+\sigma^{i j} \Pi_{i} t_{j}\right) \phi_{\perp \tau t_{0},-\mathbf{t}}^{\prime}\left(x^{\prime}\right)=s_{+} M\left(\gamma^{0} t_{0}-\gamma \cdot \mathbf{t}\right) \chi_{\perp \tau t_{0},-\mathbf{t}}^{\prime}\left(x^{\prime}\right) \\
& \left(-\sigma^{0 i} \Pi_{0} t_{i}-\sigma^{i 0} \Pi_{i} t_{0}+\sigma^{i j} \Pi_{i} t_{j}\right) \chi_{\perp \tau t_{0},-\mathbf{t}}^{\prime}\left(x^{\prime}\right)=s_{-}\left(\gamma^{0} t_{0}-\gamma \cdot \mathbf{t}\right)\left(i \partial_{\tau}+e a_{\tau}\right) \phi_{\perp \tau t_{0},-\mathbf{t}}^{\prime}\left(x^{\prime}\right)
\end{aligned}
$$

Multiplying by $\gamma^{0}$ from the left we get back the original form of equations

$$
\begin{aligned}
(\sigma \Pi t) \gamma^{0} \phi_{\perp \tau t_{0},-\mathbf{t}}^{\prime}\left(x^{\prime}\right) & =s_{+} M(\gamma \cdot t) \gamma^{0} \chi_{\perp \tau t_{0},-\mathbf{t}}^{\prime}\left(x^{\prime}\right) \\
(\sigma \Pi t) \gamma^{0} \chi_{\perp \tau t_{0},-\mathbf{t}}^{\prime}\left(x^{\prime}\right) & =s_{-}(\gamma \cdot t)\left(i \partial_{\tau}+e a_{\tau}\right) \gamma^{0} \phi_{\perp \tau t_{0},-\mathbf{t}}^{\prime}\left(x^{\prime}\right)
\end{aligned}
$$

and the wave functions transform (up to a phase) as

$$
\begin{aligned}
& \phi_{\perp \tau t}^{P}(x)=\gamma^{0} \phi_{\perp \tau t_{0},-\mathbf{t}}\left(x^{0},-\mathbf{x}\right) \\
& \chi_{\perp \tau t}^{P}(x)=\gamma^{0} \chi_{\perp \tau t_{0},-\mathbf{t}}\left(x^{0},-\mathbf{x}\right)
\end{aligned}
$$

where the superscript $P$ denotes parity. The same is true for the charged longitudinal equations of motion, where we find the wave functions transforming as

$$
\begin{aligned}
\phi_{\| \tau t}^{P}(x) & =\gamma^{0} \phi_{\| \tau t_{0},-\mathbf{t}}\left(x^{0},-\mathbf{x}\right) \\
\chi_{\| \tau t}^{P}(x) & =\gamma^{0} \chi_{\| \tau t_{0},-\mathbf{t}}\left(x^{0},-\mathbf{x}\right)
\end{aligned}
$$

The solutions of the transverse equations are constructed from a helicity projection operator, and a $\zeta_{\perp}^{ \pm}(t)$ part. A similar situation exists for the longitudinal equations. We check both components.

When we check the action of parity on the $\zeta_{\perp}^{ \pm}(t)$ parts of the solutions of the transverse equation, we find

$$
\begin{aligned}
P \zeta_{\perp}^{+}(t) \equiv \gamma^{0} \zeta_{\perp}^{+}\left(t_{0},-\mathbf{t}\right) & =\zeta_{\perp}^{-}(t) \\
P \zeta_{\perp}^{-}(t) \equiv \gamma^{0} \zeta_{\perp}^{-}\left(t_{0},-\mathbf{t}\right) & =\zeta_{\perp}^{+}(t)
\end{aligned}
$$

and

$$
\begin{aligned}
& P \eta_{\perp}^{+}(t) \equiv \gamma^{0} \eta_{\perp}^{+}\left(t_{0},-\mathbf{t}\right)=\eta_{\perp}^{-}(t) \\
& P \eta_{\perp}^{-}(t) \equiv \gamma^{0} \eta_{\perp}^{-}\left(t_{0},-\mathbf{t}\right)=\eta_{\perp}^{+}(t)
\end{aligned}
$$


On the other hand, for the solutions of the longitudinal equations, we find

$$
\begin{aligned}
& P \zeta_{\|}^{+}(t) \equiv \gamma^{0} \zeta_{\|}^{+}\left(t_{0},-\mathbf{t}\right)=\eta_{\|}^{+}(t) \\
& P \zeta_{\|}^{-}(t) \equiv \gamma^{0} \zeta_{\|}^{-}\left(t_{0},-\mathbf{t}\right)=\eta_{\|}^{-}(t)
\end{aligned}
$$

and

$$
\begin{aligned}
& P \eta_{\|}^{+}(t) \equiv \gamma^{0} \eta_{\|}^{+}\left(t_{0},-\mathbf{t}\right)=\zeta_{\|}^{+}(t) \\
& P \eta_{\|}^{-}(t) \equiv \gamma^{0} \eta_{\|}^{-}\left(t_{0},-\mathbf{t}\right)=\zeta_{\|}^{-}(t)
\end{aligned}
$$

We can make a combination of states for which this transformation makes them transfrom one into the other. This combination is useful also for charge conjugation and $\tau$ reversal. The combination is

$$
\begin{aligned}
& P\left(\zeta_{\|}^{+}(t) \pm \eta_{\|}^{-}(t)\right)=\left(\zeta_{\|}^{-}(t) \pm \eta_{\|}^{+}(t)\right) \\
& P\left(\zeta_{\|}^{-}(t) \pm \eta_{\|}^{+}(t)\right)=\left(\zeta_{\|}^{+}(t) \pm \eta_{\|}^{-}(t)\right)
\end{aligned}
$$

Doing the same thing for the transverse case one obtaines

$$
\begin{aligned}
& P\left(\zeta_{\perp}^{+}(t) \pm \eta_{\perp}^{-}(t)\right)=\left(\zeta_{\perp}^{-}(t) \pm \eta_{\perp}^{+}(t)\right) \\
& P\left(\zeta_{\perp}^{-}(t) \pm \eta_{\perp}^{+}(t)\right)=\left(\zeta_{\perp}^{+}(t) \pm \eta_{\perp}^{-}(t)\right)
\end{aligned}
$$

On the other hand, when we check the action of normal parity on the helicity projection operator part of the solutions, we find

$$
\gamma^{0} P_{h \pm}\left(p_{0},-\mathbf{p}, t_{0},-\mathbf{t}\right)=P_{h \mp}(p, t) \gamma^{0}
$$

therefore the helicity flips sign.

Another type of parity is what we call generalized parity, denoted by $\mathcal{P}$, including the regular parity and a time inversion. Usually the discrete symmetry under time inversion is treated separately and differently from that of parity, as an anti-hermitian operator. In our theory time is just another dimension distinguished from the space dimensions by the metric tensor, and the evolution is governed by $\tau$. Therefore the role of time as described by Wigner is transferred to $\tau$, the time symmetry becoming much simpler. This generalized parity transformation is

$$
x_{\mu} \rightarrow-x_{\mu}
$$




$$
\begin{aligned}
P_{\mu} & \rightarrow-P_{\mu} \\
t_{\mu} & \rightarrow-t_{\mu} \\
a_{\mu} & \rightarrow-a_{\mu}
\end{aligned}
$$

When we come to define the way the wave functions transform we have a few options. The charged form of Eqs. (64), 65)

$$
\begin{aligned}
& (\gamma \cdot t)(\sigma \Pi t) \phi_{\perp}=s_{+} M \chi_{\perp} \\
& (\gamma \cdot t)(\sigma \Pi t) \chi_{\perp}=2 s_{-}\left(i \partial_{\tau}+e a_{\tau}\right) \phi_{\perp}
\end{aligned}
$$

permits two compensating operators for the transformation of Eqs. (269), $\gamma^{5}$, and $(\gamma \cdot t)$. We prefer to use $\gamma^{5}$ as a generalized parity operator because it is not coupled to any four-vector. Applying the transformation we find

$$
\begin{aligned}
& -(\gamma \cdot t)(\sigma \Pi t) \phi_{\perp \tau,-t}^{\prime}\left(x^{\prime}\right)=s_{+} M \chi_{\perp \tau,-t}^{\prime}\left(x^{\prime}\right) \\
& -(\gamma \cdot t)(\sigma \Pi t) \chi_{\perp \tau,-t}^{\prime}\left(x^{\prime}\right)=2 s_{-}\left(i \partial_{\tau}+e a_{\tau}\right) \phi_{\perp \tau,-t}^{\prime}\left(x^{\prime}\right)
\end{aligned}
$$

and after multiplying by $\gamma^{5}$ from the left we obtain the usual form

$$
\begin{aligned}
& (\gamma \cdot t)(\sigma \Pi t) \gamma^{5} \phi_{\perp \tau,-t}^{\prime}\left(x^{\prime}\right)=s_{+} M \gamma^{5} \chi_{\perp \tau,-t}^{\prime}\left(x^{\prime}\right) \\
& (\gamma \cdot t)(\sigma \Pi t) \gamma^{5} \chi_{\perp \tau,-t}^{\prime}\left(x^{\prime}\right)=2 s_{-}\left(i \partial_{\tau}+e a_{\tau}\right) \gamma^{5} \phi_{\perp \tau,-t}^{\prime}\left(x^{\prime}\right)
\end{aligned}
$$

In this case, the wave functions transform (up to a phase) as

$$
\begin{aligned}
& \phi_{\perp \tau t}^{\mathcal{P}}(x)=\gamma^{5} \phi_{\perp \tau,-t}(-x) \\
& \chi_{\perp \tau t}^{\mathcal{P}}(x)=\gamma^{5} \chi_{\perp \tau,-t}(-x)
\end{aligned}
$$

A similar transformation holds for the longitudinal equations of motion. As can be seen by operating on the solutions of transverse and longitudinal equations, we get

$$
\begin{aligned}
& \mathcal{P} \zeta_{\perp, \|}^{+}(t) \equiv \gamma^{5} \zeta_{\perp, \|}^{+}(-t)=+\zeta_{\perp, \|}^{+}(t) \\
& \mathcal{P} \zeta_{\perp, \|}^{-}(t) \equiv \gamma^{5} \zeta_{\perp, \|}^{-}(-t)=-\zeta_{\perp, \|}^{-}(t)
\end{aligned}
$$

and

$$
\begin{aligned}
& \mathcal{P} \eta_{\perp, \|}^{+}(t) \equiv \gamma^{5} \eta_{\perp, \|}^{+}(-t)=-\eta_{\perp, \|}^{+}(t) \\
& \mathcal{P} \eta_{\perp, \|}^{-}(t) \equiv \gamma^{5} \eta_{\perp, \|}^{-}(-t)=+\eta_{\perp, \|}^{-}(t)
\end{aligned}
$$


Therefore, the generalized parity operation on the transverse equations, brings the set of solutions into themselves.

Here too, we can use combinations which behave under generalized parity the same as shown for the regular parity, but now the behavior of the transverse and longitudinal solutions is the same. For the transverse and longitudinal cases we have

$$
\begin{aligned}
& \mathcal{P}\left(\zeta_{\perp, \|}^{+}(t) \pm \eta_{\perp, \|}^{-}(t)\right)=+\left(\zeta_{\perp, \|}^{+}(t) \pm \eta_{\perp, \|}^{-}(t)\right) \\
& \mathcal{P}\left(\zeta_{\perp, \|}^{-}(t) \pm \eta_{\perp, \|}^{+}(t)\right)=-\left(\zeta_{\perp, \|}^{-}(t) \pm \eta_{\perp, \|}^{+}(t)\right)
\end{aligned}
$$

Note that the combinations

$$
\begin{aligned}
& \zeta^{+}(t)+\eta^{-}(t) \\
& \zeta^{-}(t)-\eta^{+}(t)
\end{aligned}
$$

are pure right handed, and the combinations

$$
\begin{aligned}
& \zeta^{+}(t)-\eta^{-}(t) \\
& \zeta^{-}(t)+\eta^{+}(t)
\end{aligned}
$$

are pure left handed, for both $\perp$ and $\|$. The extended helicity operator remains unchanged under $\mathcal{P}$, so there is no helicity flip in this case.

\section{C.2 Charge conjugation}

The theory is symmetric under charge conjugation. We define the charge conjugation operation, denoted by $\mathcal{C}$, as the transformation necessary to bring the charged equations of motion to the same form only with the sign of $e$ reversed. First conjugate the equations

$$
\begin{aligned}
(\gamma \cdot t)^{*}\left(\sigma^{\mu \nu *}\left(-P_{\mu}-e a_{\mu}\right) t_{\nu}\right) \phi_{\perp \tau t}^{\prime *}\left(x^{\prime}\right) & =s_{+} M \chi_{\perp \tau t}^{\prime *}\left(x^{\prime}\right) \\
(\gamma \cdot t)^{*}\left(\sigma^{\mu \nu *}\left(-P_{\mu}-e a_{\mu}\right) t_{\nu}\right) \chi_{\perp \tau t}^{\prime *}\left(x^{\prime}\right) & =2 s_{-}\left(-i \partial_{\tau}+e a_{\tau}\right) \phi_{\perp \tau t}^{\prime *}\left(x^{\prime}\right)
\end{aligned}
$$

then make the substitution

$$
\begin{gathered}
a_{\tau} \rightarrow-a_{\tau} \\
\tau \rightarrow-\tau
\end{gathered}
$$


which is required to bring the equations to the correct form. This is consistent with the invariance of the free kinetic term of the pre-Maxwell field $\frac{1}{4} \lambda f^{\alpha \beta} f_{\alpha \beta}$. The next step is to multiply them by $\left(i \gamma^{5} \gamma^{2}\right)=\left(\gamma^{5} C \gamma^{0}\right)$ where $C=i \gamma^{2} \gamma^{0}$, because

$$
\begin{aligned}
i \gamma^{5} \gamma^{2}\left(\gamma^{\mu *}\right)\left(i \gamma^{5} \gamma^{2}\right)^{-1} & =\gamma^{\mu} \\
i \gamma^{5} \gamma^{2}\left(\sigma^{\mu \nu *}\right)\left(i \gamma^{5} \gamma^{2}\right)^{-1} & =-\sigma^{\mu \nu}
\end{aligned}
$$

One then obtains

$$
\begin{aligned}
(\gamma \cdot t)(\sigma \tilde{\Pi} t) i \gamma^{5} \gamma^{2} \phi_{\perp-\tau t}^{*}\left(x^{\prime}\right) & =s_{+} M i \gamma^{5} \gamma^{2} \chi_{\perp-\tau t}^{*}\left(x^{\prime}\right) \\
(\gamma \cdot t)(\sigma \tilde{\Pi} t) i \gamma^{5} \gamma^{2} \chi_{\perp-\tau t}^{*}\left(x^{\prime}\right) & =2 s_{-}\left(i \partial_{\tau}-e a_{\tau}\right) i \gamma^{5} \gamma^{2} \phi_{\perp-\tau t}^{*}\left(x^{\prime}\right)
\end{aligned}
$$

where $\tilde{\Pi}_{\mu}=P_{\mu}+e a_{\mu}$. Taking $a_{\alpha} \rightarrow-a_{\alpha}$ brings us back to the original form. Therefore, the wave functions transform (up to a phase) as

$$
\begin{aligned}
& \phi_{\perp \tau t}^{\mathcal{C}}(x)=i \gamma^{5} \gamma^{2} \phi^{*}{ }_{\perp,-\tau, t}(x) \\
& \chi_{\perp \tau t}^{\mathcal{C}}(x)=i \gamma^{5} \gamma^{2} \chi_{\perp,-\tau, t}^{*}(x)
\end{aligned}
$$

The same persists for the longitudinal equations. This transformation includes a $\tau$ reversal for its consistency. When the pre-Maxwell field radiation equations [36] are taken into consideration,

$$
\begin{aligned}
-\partial_{\tau} f^{\tau \mu}+\partial_{\nu} f^{\mu \nu} & =e j^{\mu} \\
\partial_{\mu} f^{\tau \mu}=e j^{\tau} & =e \rho
\end{aligned}
$$

a symmetry breakdown is observed in the second equation due the need to change the sign of the $a_{\tau}$ field. This is a consequence of the Schrödinger type equation used. Here $\rho$ is a scalar. One could think of a Klein-Gordon generalization for which $j_{\tau} \propto \psi^{*} \grave{\partial}_{\tau} \psi$ for which this problem would not occur (note that the same question arises in the nonrelativistic Schrödinger quantum theory in interaction with the standard Maxwell field).

To see the consequences of performing charge conjugation to the solutions of the transverse equation, we define solutions as in Eq. (231), using $\eta_{\perp}^{r}(t)$,

$$
w_{\perp}^{r s}(p, t)=\frac{1}{2}\left(\mathbf{1}+\epsilon_{s} \frac{i}{\left|p_{\perp}\right|}(\sigma p t) \gamma^{5}\right) \eta_{\perp}^{r}(t)
$$


The subscript $s$ in Eq. (285) denotes the eigenvalue of the extended helicity operator. Considering a free event, we have for the helicity projection operator

$$
i \gamma^{5} \gamma^{2} P_{h \pm}^{*}=P_{h \mp} i \gamma^{5} \gamma^{2}
$$

and since

$$
\begin{gathered}
-i \sigma^{2} \xi^{+*}=-\xi^{-} \\
-i \sigma^{2} \xi^{-*}=+\xi^{+}
\end{gathered}
$$

for the $\zeta_{\perp}^{r}(t)$ part of the solutions, we have

$$
\begin{aligned}
& \mathcal{C} \zeta_{\perp}^{+}(t)=-\eta_{\perp}^{+}(t) \\
& \mathcal{C} \zeta_{\perp}^{-}(t)=+\eta_{\perp}^{-}(t)
\end{aligned}
$$

and

$$
\begin{aligned}
& \mathcal{C} \eta_{\perp}^{+}(t)=+\zeta_{\perp}^{+}(t) \\
& \mathcal{C} \eta_{\perp}^{-}(t)=-\zeta_{\perp}^{-}(t)
\end{aligned}
$$

so we can use the combination

$$
\begin{aligned}
& \mathcal{C}\left(\zeta_{\perp}^{+}(t) \pm \eta_{\perp}^{-}(t)\right)=-\left(\zeta_{\perp}^{-}(t) \pm \eta_{\perp}^{+}(t)\right) \\
& \mathcal{C}\left(\zeta_{\perp}^{-}(t) \pm \eta_{\perp}^{+}(t)\right)= \\
& =\left(\zeta_{\perp}^{+}(t) \pm \eta_{\perp}^{-}(t)\right)
\end{aligned}
$$

These combinations have mixed eigenvalues of the chiral precedence operator, positive for $\zeta_{\perp}^{+}(t), \eta_{\perp}^{+}(t)$, and negative for $\zeta_{\perp}^{-}(t), \eta_{\perp}^{-}(t)$. Therefore for the combination we obtain

$$
\mathcal{C} e^{-i \frac{p_{\perp}^{2}}{2 M} \tau+i p x}\left(u_{\perp}^{r, s}(p, t) \pm w_{\perp}^{-r, s}(p, t)\right)=-\epsilon_{r} e^{-i \frac{p_{\perp}^{2}}{2 M} \tau-i p x}\left(w_{\perp}^{r,-s}(p, t) \pm u_{\perp}^{-r,-s}(p, t)\right)
$$

where $\epsilon_{r}$ is \pm 1 depending on value of $r$, either + or - . The state transforms into the other helicity state. Since no space inversion was performed, we get a helicity flip. Therefore, the charge conjugated wave function describes an event with opposite charge and spin, moving in the opposite direction. As mentioned in Appendix C.1 dealing with parity, these combinations are pure left or right handed spinors. For example, the charge conjugated left handed spinor is a right handed spinor with opposite charge moving in the opposite direction.

Repeating these operations for the solutions of the longitudinal equations, we find similar results, we have only to change the subscript $\perp$ to $\|$. 


\section{C.3 $\tau$ reversal}

Here we discuss $\tau$ reversal in the sense introduced by Wigner; reversing the evolution parameter we reverse the motion of the event, thus creating a current in the opposite direction. The operations needed for the $\tau$ reversal symmetry, denoted as $\mathcal{T}$, result in the same operations as those for the charge conjugation as can be seen by performing the operation

$$
\tau \rightarrow-\tau
$$

on the equations of motion. To compensate we have to take

$$
a_{\mu} \rightarrow-a_{\mu}
$$

and applying this to Eqs. (270) while taking the conjugate of the equations, we get the transformation properties of the wave functions

$$
\begin{aligned}
\phi_{\perp \tau t}^{\mathcal{T}}(x) & =\gamma^{5} \gamma^{2} \phi^{*}{ }_{\perp,-\tau, t}(x) \\
\chi_{\perp \tau t}^{\mathcal{T}}(x) & =\gamma^{5} \gamma^{2} \chi_{\perp,-\tau, t}^{*}(x)
\end{aligned}
$$

As usual, the longitudinal equations have the same transformation properties. We can repeat the same arguments given for the charge conjugation. Although the transformation looks the same as the charge conjugation one, the reason for doing it stems from Wigner's idea of time reversal. However, we obtain a charge conjugated event.

\section{C.4 $\mathcal{P C} \mathcal{T}$ invariance}

The most striking thing about these discrete transformations is the identity of the charge conjugation transformation, and $\tau$ reversal transformation in the sense of Wigner. Conceptually this is understood using Feynman diagrams. We know from Dirac's theory that a charge conjugated electron, i.e. a positron, moves backward in space-time with opposite charge. This is exactly what a $\tau$ reversal means. Seen in the four dimensional world (a projection of the trajectory on space-time at a specific $\tau$ ), a $\tau$ reversal changes the charge, direction, and helicity. Concluding that charge conjugation and $\tau$ reversal have the same consequences, a full $\mathcal{P C} \mathcal{T}$ transformation is just a generalized parity transformation. We get an event with opposite motion in space-time relative to the state before conjugation, with the same helicity and charge. The symmetry under generalized parity is a direct consequence of the manifest covariance built into the theory. 


\section{Appendix - Dirac matrices}

Our orthogonal matrix basis which satisfies the Dirac Clifford algebra is [35, 38]

$$
\begin{array}{llll}
\Gamma_{1}=1 & \Gamma_{2}=\gamma^{0} & \Gamma_{3}=i \gamma^{1} & \Gamma_{4}=i \gamma^{2} \\
\Gamma_{5}=i \gamma^{3} & \Gamma_{6}=\gamma^{0} \gamma^{1} & \Gamma_{7}=\gamma^{0} \gamma^{2} & \Gamma_{8}=\gamma^{0} \gamma^{3} \\
\Gamma_{9}=i \gamma^{2} \gamma^{3} & \Gamma_{10}=i \gamma^{3} \gamma^{1} & \Gamma_{11}=i \gamma^{1} \gamma^{2} & \Gamma_{12}=\gamma^{1} \gamma^{2} \gamma^{3} \\
\Gamma_{13}=i \gamma^{0} \gamma^{2} \gamma^{3} & \Gamma_{14}=i \gamma^{0} \gamma^{3} \gamma^{1} & \Gamma_{15}=i \gamma^{0} \gamma^{1} \gamma^{2} & \Gamma_{16}=i \gamma^{0} \gamma^{1} \gamma^{2} \gamma^{3}
\end{array}
$$

We use the following chiral representation of the Dirac matrices

$$
\gamma^{0}=\left(\begin{array}{ll}
0 & 1 \\
1 & 0
\end{array}\right) \quad \gamma^{i}=\left(\begin{array}{rc}
0 & \sigma^{i} \\
-\sigma^{i} & 0
\end{array}\right)
$$

and

$$
\gamma^{5}=\left(\begin{array}{rr}
-1 & 0 \\
0 & 1
\end{array}\right)
$$




\section{References}

[1] T.D. Newton and E.P. Wigner, Rev. Mod. Phys. 21, 400 (1949)

[2] L.P. Horwitz and C. Piron, Helv. Phys. Acta 46, 316 (1973)

[3] R.I. Arshansky and L.P. Horwitz, Found. of Phys. 15, 701 (1985)

[4] V.A. Fock, Phys. Z. Sowjetunion 12, 404 (1937)

[5] E.C.G. Stueckelberg, Helv. Phys. Acta 14, 322 (1941); 15, 23 (1942)

[6] Y. Nambu, Prog. Theor. Phys. 5, 82 (1950)

[7] J. Schwinger, Phys. Rev. 82, 664 (1951)

[8] R.P. Feynman, Phys. Rev. 80, 440 (1950); 84, 108 (1951)

[9] J.H. Cooke, Phys. Rev. 166, 1293 (1968)

[10] J.R. Fanchi, Phys. Rev. A 34, 1677 (1986)

[11] L.P. Horwitz, C. Piron and F. Reuse, Helv. Phys. Acta 48, 546 (1975)

[12] F. Reuse, Helv. Phys. Acta 51, 157 (1978)

[13] R. Kubo, Nuovo Cimento A 85, 293 (1985)

[14] W.C. Davidon, Phys. Rev. 97, 1131, 1139 (1955)

[15] P.A.M. Dirac, Proc. Roy. Soc. A117, 610 (1928); A118, 351 (1928)

[16] J.R. Fanchi, Found. of Phys. 23, 487 (1993)

[17] J.A. Wheeler and R.P. Feynman, Rev. Mod. Phys. 21, 425 (1949)

[18] C.W. Misner, S. Thorne and J.A. Wheeler, Gravitation, (W.H.Freeman \& Company, New York 1973)

[19] J.R. Fanchi, Found. of Phys. 24, 543 (1994)

[20] E.W. Otten, Nuc. Phys. B (Proc. Suppl.) 38, 26 (1995) 
[21] M.K. Moe, Nuc. Phys. B (Proc. Suppl.) 38, 36 (1995)

[22] D.W. Sciama, Nuc. Phys. B (Proc. Suppl.) 38, 320 (1995)

[23] L.P. Horwitz and R. Arshansky, J.Phys.A :Math.Gen. 15, L659-L662 (1982)

[24] J.D. Bjorken and S.D. Drell, Relativistic Quantum Mechanics, (McGraw-Hill, New York 1964)

[25] L.P. Horwitz, Found. of Phys. 22, 421 (1992)

[26] G.C. Hegerfeldt, Phys. Rev. D10, 3320 (1974); Phys. Rev. Lett. 54, 2395 (1985)

[27] L.P. Horwitz, R.I. Arshansky and A.C. Elitzur, Found. of Phys. 18, 1159 (1988)

[28] L.P. Horwitz, Found of Phys. 15, 701 (1985)

[29] M.C. Land and L.P. Horwitz, J.Phys.A :Math.Gen. 28, 3089 (1995)

[30] M.C. Land and L.P. Horwitz, Found. of Phys. 24, 563 (1994)

[31] N. Shnerb and L.P. Horwitz, Phys. Rev. A 48, 4068 (1993)

[32] A. Arensburg and L.P. Horwitz, Found. of Phys. 22, 1025 (1992)

[33] E. Wigner, Annals of Math. 40, 149 (1939)

[34] C. Itzykson and J.B. Zuber, Quantum Field Theory, (McGraw-Hill, New York 1980)

[35] S.S. Schweber, An Introduction to Relativistic Quantum Field Theory, (Harper \& Row, New York 1961)

[36] D. Saad, L.P. Horwitz and R.I. Arshansky, Found. of Phys. 19, 1125 (1989)

[37] J. Schwinger, Phys. Rev. 82, 914 (1951)

[38] B. Thaller, The Dirac Equation, (Springer-Verlag, New York 1992) 